\title{
A VARIATIONAL APPROACH FOR SHARPENING HIGH DIMENSIONAL IMAGES *
}

\author{
MICHAEL MÖLLER ${ }^{\dagger}$, TODD WITTMAN ${ }^{\ddagger}$, ANDREA L. BERTOZZI ${ }^{\ddagger}$, AND MARTIN \\ BURGER †
}

\begin{abstract}
Earth observing satellites usually not only take ordinary red-green-blue images, but provide several images including the near-infrared and infrared spectrum. These images are called multispectral, for about four to seven different bands, or hyperspectral, for higher dimensional images of up to 210 bands. The drawback of the additional spectral information is that each spectral band has rather low spatial resolution. In this paper we propose a new variational method for sharpening high dimensional spectral images with the help of a high resolution gray scale image while preserving the spectral characteristics used for classification and identification tasks. We describe the application of Split Bregman minimization to our energy, prove convergence speed and compare the Split Bregman method to a descent method based on the ideas of alternating directions minimization. Finally, we show results on Quickbird multispectral as well as on AVIRIS hyperspectral data.
\end{abstract}

Key words. image fusion, multispectral, hyperspectral, pan-sharpening, variational

1. Introduction. Instead of just taking red-green-blue images many satellite imaging systems, such as the Quickbird and Landsat-7 satellites, produce so-called multispectral images including the near-infrared spectrum and consist of four to seven bands. These additional bands can be used for various identification and classification tasks. Because images in a precise spectral range can only be taken at rather low spectral resolution, many satellites also include a so-called panchromatic image, which is a gray-scale image that spans a wide range of frequencies, but comes at high spatial resolution. Pan-sharpening is the process of fusing the low resolution multispectral image with the high resolution panchromatic image to obtain a high resolution multispectral image.

The goal of pan-sharpening is to combine the high spatial resolution of the panchromatic image with the precise spectral information of the multispectral image. The resulting image should have high visual quality to aid in detection and classification tasks. However, the pan-sharpened image should also contain the same spectral (color) information as the original multispectral data for precise identification of targets.

Several methods have been proposed for pan-sharpening multispectral imagery. Many techniques express the panchromatic image as a linear combination of the multispectral bands, including the Intensity-Hue-Saturation (IHS) ([17, 16, 44]) and Brovey methods $([22])$. Other methods project the images into a different space like Principal Component Analysis (PCA) ([42]). Several authors have proposed using the wavelet transform or other types of MRA to extract geometric edge information from the panchromatic image. These details are injected into the low resolution image,

*This work was supported by the US Department of Defense, ONR grant N000140810363, NSF grant ACI-0321917 and NSF grant DMS-0601395. MB was supported by DFG project "regularization with singular energies". Preliminary computational results on hyperspectral data were previously presented at the SPIE Conference on Algorithms and Technologies for Multispectral, Hyperspectral, and Ultraspectral Imagery XV ([33]). This paper is based on the results from the masters thesis [32].

${ }^{\dagger}$ Westfälische Wilhelms-Universität Münster, Institut für Numerische und Angewandte Mathematik, Einsteinstr. 62, D 48149 Münster, Germany, email: michaelm@math.ucla.edu, martin.burger@uni-muenster.de

${ }_{\ddagger}^{\ddagger}$ Department of Mathematics, University of California, Los Angeles, CA 90059, email: \{wittman, bertozzi\}@math.ucla.edu 
sometimes based on certain decision models ([49, 28, 31, 2]). Improvements, hybrid schemes and other methods focusing on spectral consistency have been proposed $([3,41,35,23,25])$. Recently, Ballester et. al. introduced a variational method called $\mathrm{P}+\mathrm{XS}$ image fusion that explicitly forces the edges of the pan-sharpened image to line up with those in the panchromatic image ([6]).

Several survey papers have compared the performance of pan-sharpening methods $([45,21,22,5])$. Generally, the methods producing the highest spatial quality tend to distort the spectral quality of the image. Very interesting observations have been made by Thomas et al. in [43]. One of the main points in their paper is that imposing a relationship between the remotely sensed image comes with the risk to drastically weaken the fusion performance.

Almost all methods impose a certain spectral relation between the panchromatic image and the spectral bands like the common assumption that the panchromatic image can be modeled as a linear combination of the spectral bands. As described in [43] this assumption can lead to heavy spectral distortion, since it does not reflect the physics correctly. Furthermore, these methods do not extend to higher dimensional images. For bands whose frequency is not covered by the panchromatic image their main assumption is no longer valid.

Our goal is to combine ideas from Wavelet fusion and $\mathrm{P}+\mathrm{XS}$ fusion with a new spectral consistency term into a single variational framework that does not impose any direct spectral relation between the multispectral and the panchromatic images. We should mention that our approach does not respect all possible image input characteristics described in [43], but no assumption is made on the link between the intensity values of the multispectral and the panchromatic image. In other words, the high resolution gray scale image does not have to be panchromatic, but could be any high resolution image, an image we call master image, showing the same scene as the multispectral. Therefore, our method naturally extends to higher dimensions, i.e. hyperspectral images. Despite the missing spectral relation, we still want to preserve the spectral information from the low resolution spectral image in the sharpening process. We will present two slightly different energies: One which explicitly includes matching of wavelet coefficients in the wavelet domain and an alternate energy, which can be minimized in the spatial domain only and therefore has big computational advantages. We refer to our models as variational wavelet pansharpening (VWP) and the alternate energy (AVWP).

In Section 2, we present the energy models that describes the desired qualities of the sharpened image. We discuss numerical methods for minimizing the energy in Section 3. In Section 4, we present results on multispectral Quickbird data as well as hyperspectral data which we sharpen with Google maps ${ }^{\text {TM }}$ [1] screen shots as master images. We also show to preserve the spectral information from the original data. Finally, we conclude in Section 5 by suggesting extensions of VWP and areas for future research.

A more detailed description on our image fusion method can be found in the masters thesis [32]. Preliminary results on hyperspectral data were previously presented at the SPIE Conference on Algorithms and Technologies for Multispectral, Hyperspectral, and Ultraspectral Imagery XV ([33]).

2. Energy Functional. The general idea of variational image processing methods is to develop an energy functional depending on an image, where a low value of the energy functional corresponds to a good quality image.

Many existing pan-sharpening methods like IHS, Brovey, and P+XS image fusion 
assume that the panchromatic image is a linear combination of the different bands. Looking at the spectral response of the sensors of the Quickbird satellite system, this assumption does not seem to be true in general. ([36], Fig. 1). A false linear combination assumption can lead to spectral distortion and therefore damage the spectral information of the original multispectral image ([43]). Furthermore, imposing a relation between the high resolution and the multispectral image as well as modeling the image formation process makes the method sensor dependent and restricts it to the bands whose spectral response is covered by the master image.

Therefore, we propose a method that does not depend on this assumption. The VWP energy model consists of four parts and is specifically designed to sharpen images with an arbitrary number of bands while preserving spectral quality. In the following we describe each term of our energy functional separately. We refer to the master image as $M: \Omega \rightarrow \mathbb{R}$, where $\Omega \subset \mathbb{R}^{2}$ is the image domain. $H_{i}$ are the original low resolution and $u_{i}$ the desired high resolution spectral bands.

2.1. Geometry enforcing term. The first variational pan-sharpening method was proposed by Ballester et al. in 2006 ([6]). Their idea for introducing the geometry of the panchromatic image was to align all level lines of the high resolution image with each multispectral band. The main assumption here is that the geometric information of an image is contained in its level sets, independent of their actual level.

The level sets of an image can be represented by the vector field $\theta$ consisting of all unit normal vectors of those level sets. The definition of $\theta$ is somehow problematic: On smooth parts of the image the gradient is zero and at edges a classical or a weak derivative does not exist. For practical purposes we use an additional regularization and define $\theta_{\epsilon}(x)=\frac{\nabla M(x)}{|\nabla M(x)|_{\epsilon}}$, where $|\nabla M|_{\epsilon}=\sqrt{\left(D_{x} M\right)^{2}+\left(D_{y} M\right)^{2}+\epsilon^{2}}$ is an approximation of the unit normal vector field.

For the true unit normal vector field $\theta$ we have $\theta \cdot \nabla M=|\nabla M|$. To ensure that each spectral band has the same level sets as the panchromatic image, Ballester et al. suggested to align the normal vectors of the level sets. Therefore, every band of the restored image should satisfy $\left|\nabla u_{n}\right|-\theta \cdot \nabla u_{n}=0$. Integrating over the left hand side and applying integration by parts leads to $\int_{\Omega}\left(\left|\nabla u_{n}\right|+\operatorname{div}(\theta) u_{n}\right)$. The total energy Ballester et al. suggested to minimize in [6] is

$$
\begin{aligned}
J_{P+X S}(u)= & \sum_{n=1}^{N} \int_{\Omega}\left(\left|\nabla u_{n}\right|+\operatorname{div}(\theta) u_{n}\right) d x \\
& +\lambda \int_{\Omega}\left(\sum_{n=1}^{N} \alpha_{n} u_{n}-M\right)^{2} d x \\
& +\mu \sum_{n=1}^{N} \int_{\Omega} \pi_{S}\left(\left(k * u_{n}\right)-H_{n}\right)^{2} d x,
\end{aligned}
$$

where $\lambda$ and $\mu$ are parameters, $k$ is a convolution kernel modeling the blur of the low resolution data and $\pi_{S}$ is a dirac comb indicating which multispectral pixel values are given by the low resolution data. Increasing the resolution by a factor of four the discrete dirac comb would have ones every fourth pixel and zeros elsewhere. This energy was proposed for sharpening multispectral images with $M$ being a high resolution panchromatic image. The second term reflects the assumption that $M$ is a linear combination of the spectral bands $u_{n}$ with mixing coefficients $\alpha_{n}$.

The idea for introducing the geometry of a higher resolution image can easily be adapted for the sharpening of hyperspectral images with an arbitrary master image 
at high resolution. $\theta$ depends only on the level sets of the image and therefore no assumption on the relation between the hyperspectral image and the master image needs to be made. In particular, the method is independent of contrast and illumination changes in the master image.

We start the construction of our energy similarly to the P+XS model. As discussed above, introducing the geometric information from the master image means minimizing the quantity $\left|\nabla u_{n}\right|-\theta \cdot \nabla u_{n}$ over the whole image domain $\Omega$. Integration by parts leads to

$$
\hat{E}_{g}=\gamma \sum_{n=1}^{N}\left[\int_{\Omega}\left|\nabla u_{n}\right| d x+\int_{\Omega} \operatorname{div}(\theta) \cdot u_{n} d x\right]
$$

The idea of aligning the gradient vectors of an image with a smooth unit normal vector field similar to (2.2) was also proposed by Lysaker, Osher and Tai ([29]) for a new image denoising algorithm and led to iterative regularization using the Bregman distance ([34]). Therefore, it is not surprising that the geometry introducing term (2.2) also has a link to Bregman distances. In the next subsection we will briefly recall the concept of Bregman distances and show the connection to the contour alignment term $(2.2)$.

2.1.1. Bregman distance and its link to the alignment of isocontours. The (generalized) Bregman distance associated with a convex energy functional $J$ is defined as

$$
D_{J}(u, v)=\{J(u)-J(v)-\langle p, u-v\rangle \mid p \in \partial J(u)\}
$$

where $\partial J(u)$ denotes the subdifferential of $J$ at $u$. While for a Frechet differentiable functional the Bregman distance is unique, it can be multivalued for non differentiable functionals like the total variation. Each element $D_{J}^{p}(u, v)$ represents a distance in the sense that $D_{J}^{p}(u, v) \geq 0$. For a strictly convex $J$ we even get $D_{J}^{p}(u, v)>0$ for $u \neq v$.

Bregman distances were originally introduced in [7] as a functional analytical concept to find extrema of convex funtionals. Recently, many papers have made use of Bregman distances to develop new image processing and optimization algorithms and prove convergence rates (e.g. $[34,8,24,10])$.

It turns out that in the case of the geometry enforcing term from $\mathrm{P}+\mathrm{XS},(2.2)$, we are also minimizing a Bregman distance: For a smooth master image with nonzero gradient $-\operatorname{div}(\theta)=-\operatorname{div}\left(\frac{\nabla M}{|\nabla M|}\right)$ is the subgradient of the TV semi-norm at $M$. Hence, we know that $|M|_{T V}=\langle-\operatorname{div}(\theta), M\rangle$. Adding zero in the form of $-|M|_{T V}+$ $\langle\operatorname{div}(\theta),-M\rangle$ to term $(2.2)$ then gives us

$$
\begin{aligned}
\hat{E}_{g} & =\gamma \sum_{n=1}^{N}\left(\left|u_{n}\right|_{T V}-|M|_{T V}+\left\langle\operatorname{div}(\theta), u_{n}-M\right\rangle\right) \\
& =\gamma \sum_{n=1}^{N} D_{|\cdot|_{T V}}^{-\operatorname{div}(\theta)}\left(u_{n}, M\right),
\end{aligned}
$$

which shows that aligning the isocontours of each multispectral band with the master image is nothing but minimizing their Bregman distance with respect to the TV seminorm. We penalize derivations from the master image in the Bregman distance. 
It is shown in [39] that Bregman distance regularization with respect to TV is linked to texture enhancement. Furthermore, the Bregman distance is contrast invariant. This indicates that this type of regularization is very well suited for our problem because we are "close" to the master image independent of the master images actual values, which means that the edges and jumps in the image must be in the right places.

2.2. Contrast Increasing Contour Alignment. Despite the nice link to Bregman distances we shall also consider a different weighting of the two terms in the energy functional and write

$$
E_{g}=\sum_{n=1}^{N}\left[\gamma \int_{\Omega}\left|\nabla u_{n}\right| d x+\eta \int_{\Omega} \operatorname{div}(\theta) \cdot u_{n} d x\right] .
$$

The introduction of the additional parameter $\eta$ might not be immediately clear since it slightly contradicts the derivation of this term i.e. that ideally we have $\left|\nabla u_{n}\right|-$ $\theta \cdot \nabla u_{n}=0$. We found during our numerical experiments that choosing a slightly higher parameter $\eta>\gamma$ gives better spatial quality. In the next paragraph we will look at the meaning of using different parameters in a mathematical sense and further motivate our decision by some simple one dimensional examples.

The optimality condition for minimizing the functional (2.5) is

$$
\operatorname{div}\left(\frac{\nabla u}{|\nabla u|}\right)=\frac{\eta}{\gamma} \operatorname{div}(\theta) .
$$

First of all note that the divergence of the normals (a quantity of the form $\operatorname{div} \frac{\nabla a}{|\nabla a|}$ ) has a geometric meaning, i.e. the mean curvature of the level lines. The optimality condition yields that for $\eta=\gamma$ we enforce the curvature of our desired image $u$ to be the same as in our high resolution master image $M$. If we now choose $\eta$ to be greater than $\gamma$, we enforce the curvature of the desired image $u$ to be even higher. The desired curvature is the one of the panchromatic image multiplied by $\frac{\eta}{\gamma}>1$. To see the effect of a higher curvature we implemented an evolution of the form

$$
u^{k+1}=u^{k}+\tau\left(\operatorname{div}\left(\frac{\nabla u^{k}}{\left|\nabla u^{k}\right|}\right)-\frac{\eta}{\gamma} \operatorname{div}(\theta)\right)
$$

for some example signals in one dimension where we took the data as our initialization $u_{0}$ and ran (2.7) for a limited number of iterations. The results are shown in Figure 2.1 .

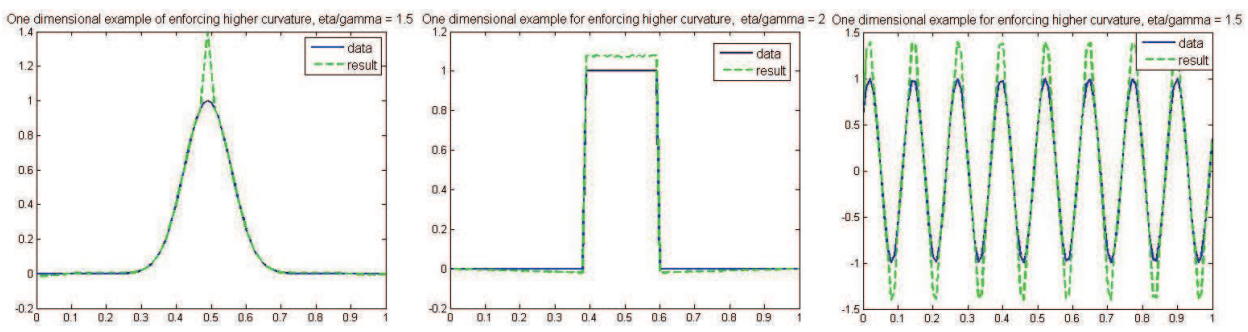

FIG. 2.1. Examples of enforcing higher curvature. Left: Gaussian signal, middle: step function, right: sinusoidal signal

One can see that all results (green curves) have a higher contrast than the original signals. The peaks and parts of high curvatures have larger values after the evolution. 
For the second example, the step function, the result can be proved analytically even for higher dimensions.

In image denoising regularization by total variation is known to decrease the contrast of images, while the our weighting increases the contrast. Maybe the two effects cancel out such that in sum a better, more realistic image is obtained.

2.3. Fidelity terms. To preserve as much spectral information as possible we would like our fused spectral image to be very close to the low resolution image on those parts of the image, where there are no edges or texture. On the edges we would like to increase the contrast and enhance the spatial information. Therefore, we decided to include a matching term in the wavelet domain.

A second level undecimated wavelet decomposition of the master image and each spectral band is calculated. A well known concept in wavelet pan-sharpening is to use the detail coefficients of the high resolution image (most likely containing the geometric information) and the approximation coefficients of each multispectral band (containing the color or spectral information) to obtain a sharpened image via the reconstruction from these coefficients ([49]). This concept is illustrated in Figure 2.2 for a stationary wavelet transformation.

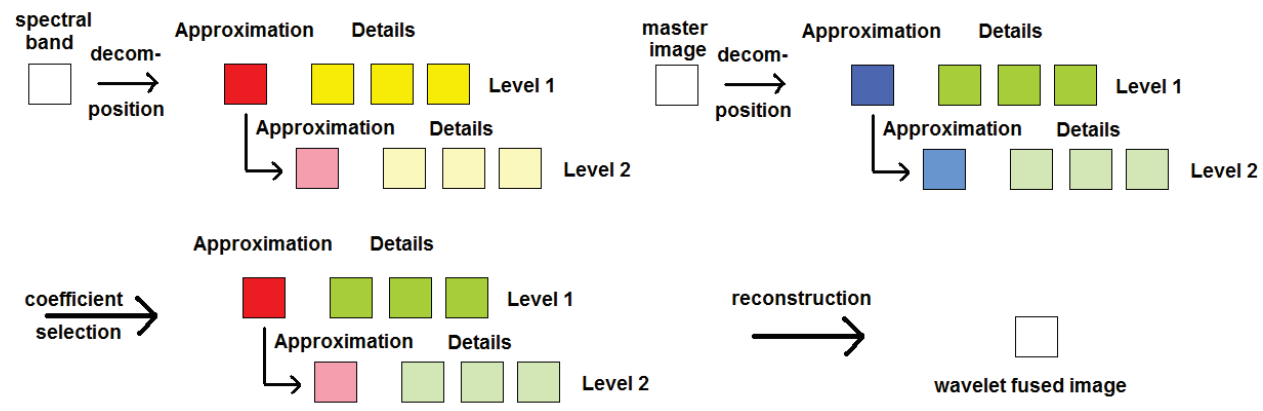

FIG. 2.2. Concept of the wavelet pan-sharpening

Despite the higher computational costs as well as memory requirements stationary wavelets have proved to be superior to the orthogonal wavelet transform because they are translational invariant and therefore much more robust towards slightly inaccurate registration and aliasing effects $([15,36,26,2])$.

In our energy we would like to be able to weight the importance of the wavelet coefficient matching differently for different levels of decomposition and type of coefficients. Depending on the desired image features the matching of the detail coefficients from the high resolution image could be more or less important than the approximation coefficient matching to the low resolution image.

To formalize the wavelet matching in a mathematical context for our energy functional let us briefly recall the wavelet notation. For a one dimensional wavelet transform, let $\phi$ be a scaling function and $\psi$ the corresponding wavelet generating a wavelet orthonormal basis of $L^{2}(\mathbb{R})$. We define the wavelets $\psi^{1}(x)=\psi\left(x_{1}\right) \phi\left(x_{2}\right)$, $\psi^{2}(x)=\phi\left(x_{1}\right) \psi\left(x_{2}\right), \psi^{3}(x)=\psi\left(x_{1}\right) \psi\left(x_{2}\right)$ and denote for $1 \leq k \leq 3, j \in \mathbb{Z}$ and $n=\left(n_{1}, n_{2}\right) \in \mathbb{Z}^{2}$

$$
\psi_{j, n}^{k}(x)=\frac{1}{2^{j}} \psi^{k}\left(\frac{x_{1}-2^{j} n_{1}}{2^{j}}, \frac{x_{2}-2^{j} n_{2}}{2^{j}}\right) .
$$


Further we define a two dimensional scaling function by

$$
\phi_{j, n}^{2}(x)=\frac{1}{2^{j}} \phi\left(\frac{x_{1}-2^{j} n_{1}}{2^{j}}\right) \phi\left(\frac{x_{2}-2^{j} n_{2}}{2^{j}}\right) .
$$

Then the approximation coefficients of a two-dimensional function are given by the scalar product with $\phi^{2}$ and the three detail coefficients (which can be seen as horizontal, vertical and diagonal details) are given by the scalar product with $\psi^{k}, k \in\{1,2,3\}$. We define the approximation matching coefficients for band $i$ as

$$
a_{j}^{i}[n]=\left\langle\uparrow H_{i}, \phi_{j, n}^{2}\right\rangle,
$$

where $\uparrow H_{i}$ denotes upsampling of the low resolution spectral band. In our experiments we used bilinear interpolation. The matching detail coefficients are taken from the scalar product with the panchromatic image and are equal for all different bands,

$$
d_{\{k, j\}}[n]=\left\langle P, \psi_{j, n}^{k}\right\rangle, \text { for } 1 \leq k \leq 3 .
$$

If we denote the desired approximation coefficients for band $i$ by $\alpha_{j}^{i}[n]$ and the desired detail coefficients by $\beta_{\{k, j\}}^{i}[n]$ then we add the following term to our energy functional

$$
\begin{aligned}
E_{w}= & \sum_{n} c_{0}\left(a_{L}^{i}[n]-\alpha_{L}^{i}[n]\right)^{2} \phi_{j, n}^{2}(x) \\
& +\sum_{n} \sum_{j=1}^{L} \sum_{k=1}^{3} c_{j}\left(d_{\{k, j\}}[n]-\beta_{\{k, j\}}^{i}[n]\right)^{2} \psi_{j, n}^{k}(x),
\end{aligned}
$$

where $c_{0}$ is the parameter for the approximation coefficient matching, $c_{j}, 1 \leq j \leq L$ are the parameters for the different levels of detail coefficient matching, and $L$ is the level of decomposition. In our experiments we used $L=2$. Notice that we assumed that our continuous representations of the images are elements of $V_{0}^{2}=\operatorname{span}\left(\phi_{0, n}^{2}\right)$ since this holds for the discrete formulation anyway. In our variational context we choose the parameters according to the type of image we would like to produce. For high spatial quality we want to introduce the edge information of the panchromatic image and therefore increase $c_{1}$ and $c_{2}$. Vice-versa we would choose larger values for $c_{0}$ for better spectral quality.

A second matching term we introduce is based on the assumption that the best spectral information can be obtained from the low resolution image. Therefore, homogeneous parts without edges or texture should be contained as they are. However, the set of edges and texture is easy to get from the master image. Unlike segmentation methods (like Mumford-Shah), where the edge set is an unknow we can simply calculate it in the master image. To detect edges and texture we apply the exponential edge detector $\exp \left(-\frac{d}{|\nabla M|^{2}}\right)$ to our master image $M$ (with an appropriate constant $d$, e.g. $d=0.004)$. We add

$$
E_{c}=\nu \sum_{i=1}^{N} \int_{\Omega \backslash \Gamma}\left(u_{i}-\uparrow H_{i}\right)^{2} d x
$$

to our energy functional.

The combination of terms in the spatial domain and in the wavelet domain has been applied in various image processing applications (e.g. $[13,19,30])$. Except for the 
advantage that one can choose different weights in the wavelet matching to obtain a certain type of image this kind of combination also has a drawback. Each minimization method will have to alternate between the spatial domain and the wavelet domain to include the different types of terms our energy functional contains. Using stationary wavelets this procedure can become extremely time as well as memory consuming, particularly for very high dimensional images with up to 210 bands. Therefore, we developed an alternate energy by choosing another matching term, that approximates the two terms (2.13) and (2.12) from above.

If we choose the wavelet matching weights equal, $c_{0}=c_{1}=c_{2}$, the above term becomes a least squares match to the wavelet fused image. Respecting the idea that the best information we have away from the edges is the low resolution image, we construct a new matching image as a combination between the low resolution and the wavelet fused image. Denoting the wavelet fused image for the $n^{\text {th }}$ band with $W_{n}$ we define the new matching image $Z_{n}$ to be

$$
Z_{n}=\exp \left(-\frac{d}{|\nabla M|^{2}}\right) \cdot W_{n}+\left(1-\exp \left(-\frac{d}{|\nabla M|^{2}}\right)\right) \cdot \uparrow H_{n}
$$

We then force our result to be close to the fused image $Z_{n}$ by adding the term

$$
E_{a}=\nu \sum_{n=1}^{N} \int_{\Omega}\left(u_{n}-Z_{n}\right)^{2} d x
$$

to our energy.

2.4. Spectral correlation preserving term. The previous terms constrain the colors within each band and introduce spatial information from the master image. The main focus was the spatial enhancement of the spectral image. However, the great use of hyperspectral images is the possibility to identify materials based on the spectral information in the image. For this application it is common to look at the spectral z-direction at each pixel (see Figure 2.3). Most classification methods are not so much based on the magnitude but on the shape of the spectral signature, i.e. the signal in z-direction, at each pixel. Hence, it is crucial to preserve this signature in the sharpening process which means we have to introduce a coupling term that preserve the relation between the bands pixel by pixel.

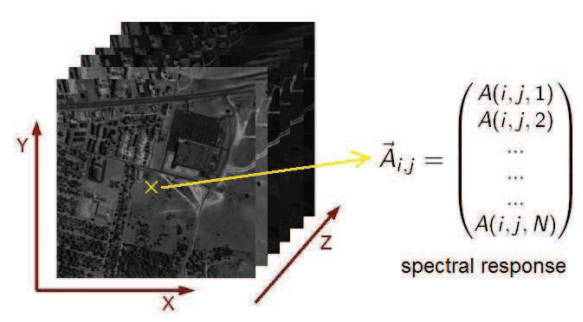

FiG. 2.3. Common viewpoint for spectral response analysis

To sharpen the image we have to allow the magnitude of the spectral signature to change. However, we want to preserve its shape. Instead of constraining the spectral signature itself we propose to keep every possible ratio of two different spectral bands of our sharpened image equal to the ratio of the same bands of the original hyperspectral image. Mathematically, this means that we would like to obtain $\frac{u_{i}}{u_{j}}=$ 
$\frac{\uparrow H_{i}}{\uparrow H_{j}} \Rightarrow u_{i} \cdot \uparrow H_{j}-u_{j} \cdot \uparrow H_{i}=0$ at every pixel. We add the sum of the squares of the corresponding $L_{2}$ norms to our energy functional:

$$
E_{s}=\mu \sum_{i, j=1, i<j}^{N} \int_{\Omega}\left(u_{i} \cdot \uparrow H_{j}-u_{j} \cdot \uparrow H_{i}\right)^{2} d x
$$

For any spectral signature $\vec{a}$ in the low resolution image and a corresponding signature $\vec{b}$ in the high resolution image, the above energy term $E_{s}$ reaches its minimum for $a(i) \cdot b(j)-a(j) \cdot b(i)=0 \forall i, j$. For $b_{j} \neq 0$ we can write this as $a(i)=\frac{a(j)}{b(j)} \cdot b(i)=0 \forall i, j$ and see that $\vec{a} \| \vec{b}$. The spectral angle, $S A M=\arccos \left(\frac{\langle\vec{a}, \vec{b}\rangle}{\|\vec{a}\| \cdot\|\vec{b}\|}\right)$, is a widely used metric to compare and evaluate spectral signatures $([48,22,5])$. Notice that the spectral correlation preserving term $E_{s}$ tries to keep the spectral vectors parallel and therefore minimizes the spectral angle. In the minimum of (2.16) all classification methods based on SAM will give the same result on the sharpened image as they do on the original spectral image.

We constructed two different energies. The VWP energy combining wavelet and spatial terms

$$
E_{V W P}(u)=E_{g}+E_{s}+E_{w}+E_{c}
$$

and the alternate energy (AVWP) which can be minimized in the spatial domain only. Putting the terms (2.5), (2.15) and (2.16) together, the total energy functional is

$$
E(u)=E_{g}+E_{s}+E_{a}
$$

In this paper, we will focus on the results produced by the latter energy. We can show that there exists a minimizer for this energy functional and the minimizer is unique. For the proof of existence and uniqueness as well as for a comparison of the different methods we refer to [32].

To practically minimize (2.18) we compared two approaches. First we use gradient descent and discretize the resulting PDE semi-implicitly similar to the ideas for alternating direction minimization (ADI) introduced by Douglas, Peaceman and Rachford $([37,20])$. The second approach uses the more recent technique of "Split Bregman" proposed by Goldstein and Osher ([24]).

\section{Numerical Minimization.}

3.1. ADI minimization. The ADI approach has been used in image processing and for minimizing total variation related functionals before (e.g. [12, 27, 11]). We make use of the necessary condition for minima that the first variation of the energy functional must equal zero. Unfortunately, the total variation part of the energy is not differentiable so we need to use subdifferential calculus or an additional smoothing similar to the epsilon regularization we used in the calculation of $\theta_{\epsilon}$. We replace $\left|\nabla u_{n}\right|$ 
by $\left|\nabla u_{n}\right|_{\epsilon}$ and obtain for the n-th band

$$
\begin{aligned}
& \frac{\delta E_{g}}{\delta u_{n}}=-\left(\gamma \operatorname{div}\left(\frac{\nabla u_{n}}{\left|\nabla u_{n}\right|_{\epsilon}}\right)-\eta \operatorname{div}\left(\theta_{\epsilon}\right)\right) . \\
& \frac{\delta E_{s}}{\delta u_{n}}=2 \mu \sum_{j=1, j \neq n}^{N}\left(u_{n} \cdot \uparrow H_{j}-u_{j} \cdot \uparrow H_{n}\right) \uparrow H_{j} . \\
& \frac{\delta E_{c}}{\delta u_{n}}=2 \nu\left(u_{n}-Z_{n}\right) .
\end{aligned}
$$

The optimality condition

$$
\frac{\delta E_{g}}{\delta u_{n}}+\frac{\delta E_{s}}{\delta u_{n}}+\frac{\delta E_{c}}{\delta u_{n}}=0
$$

can be solved numerically by introducing an artificial time variable and solving

$$
\frac{d}{d t} u_{n}=-\left(\frac{\delta E_{g}}{\delta u_{n}}+\frac{\delta E_{s}}{\delta u_{n}}+\frac{\delta E_{c}}{\delta u_{n}}\right)
$$

to steady state. In our implementation we discretize in time implicitly for $\frac{\delta E_{s}}{\delta u_{n}}$ and $\frac{\delta E_{c}}{\delta u_{n}}$. For $\frac{\delta E_{g}}{\delta u_{n}}$ we lag the denominator by one time step (for not having to solve a nonlinear equation each time step) and then follow the ideas of ADI minimization. We first take a step implicitly for the derivative in $\mathrm{x}$-direction and explicitly for the derivative in $y$-direction and then change. This leaves us with a linear system where the corresponding matrix is tridiagonal such that each step can be solved extremely fast e.g. by the Thomas (TDMA) algorithm ([18]).

3.2. Split Bregman. The Split Bregman method was recently proposed by Goldstein and Osher and is a fast minimization scheme for TV- or generally $L_{1^{-}}$ related energies. Before the calculation of the Euler-Lagrange equation a new variable is introduced to split the non-differentiable TV part from the rest of the energy. This way no additional $\epsilon$ regularization as for the ADI method is needed. More specific, minimizing $(2.18)$ is equivalent to

$$
\min _{u_{n}, d_{n}} E\left(u_{n}, d_{n}\right)=\min _{u_{n}, d_{n}} \gamma\left|d_{n}\right|_{L_{1}}+R\left(u_{n}\right) \text { such that } d_{n}=\nabla u_{n},
$$

where $R\left(u_{n}\right)$ denotes the energy functional without the total variation term:

$$
\begin{aligned}
R\left(u_{n}\right)= & \nu \int_{\Omega}\left(u_{n}-Z_{n}\right)^{2} d x \\
& +\mu \sum_{j=1, j \neq n}^{N} \int_{\Omega}\left(u_{n} \cdot \uparrow H_{j}-u_{j} \cdot \uparrow H_{n}\right)^{2} d x \\
& +\eta \int_{\Omega} \operatorname{div}(\theta) \cdot u_{n} d x .
\end{aligned}
$$

It is shown in [24] with the help of the convergence results in [34] that a constrained minimization problem like (3.6) can be solved by using the Lagrangian form

$$
\min _{u_{n}, d_{n}} \gamma\left|d_{n}\right|_{L_{1}}+R\left(u_{n}\right)+\frac{\lambda}{2}\left\|d_{n}-\nabla u_{n}\right\|_{2}^{2}
$$


and now instead of letting $\lambda$ go to infinity (a common approach), apply the Bregman iteration

$$
\begin{aligned}
\left(u_{n}^{k+1}, d_{n}^{k+1}\right) & =\underset{u_{n}, d_{n}}{\operatorname{argmin}} D_{E}^{p}\left(u_{n}, u_{n}^{k}, d_{n}, d_{n}^{k}\right)+\frac{\lambda}{2}\left\|d_{n}-\nabla u_{n}\right\|_{2}^{2} \\
& =\underset{u_{n}, d_{n}}{\operatorname{argmin}} E\left(u_{n}, d_{n}\right)-\left\langle p_{u}^{k}, u_{n}-u_{n}^{k}\right\rangle-\left\langle p_{d}^{k}, d_{n}-d_{n}^{k}\right\rangle+\frac{\lambda}{2}\left\|d_{n}-\nabla u_{n}\right\|_{2}^{2}, \\
p_{u}^{k+1} & =p_{u}^{k}-\lambda \nabla^{T}\left(\nabla u_{n}^{k+1}-d_{n}^{k+1}\right), \\
p_{d}^{k+1} & =p_{d}^{k}-\lambda\left(d_{n}^{k+1}-\nabla u_{n}^{k+1}\right) .
\end{aligned}
$$

This rather complicated formulation can be carried out in a simple two-step procedure without having to use the subgradients $p_{u}$ and $p_{d}$. The scheme (3.9) is equivalent to the Uzawa-type algorithm

$$
\begin{aligned}
\left(u_{n}^{k+1}, d_{n}^{k+1}\right) & =\left.\underset{u_{n}, d_{n}}{\operatorname{argmin} \gamma \mid} \sqrt{d_{x}^{2}+d_{y}^{2}}\right|_{L_{1}}+H(u)+\frac{\lambda}{2}\left\|d_{n}-\nabla u_{n}-b_{n}^{k}\right\|_{2}^{2}, \\
b_{n}^{k+1} & =b_{n}^{k}+\left(\nabla u_{n}^{k+1}-d_{n}^{k+1}\right) .
\end{aligned}
$$

The optimization for $u_{n}^{k+1}$ and $d_{n}^{k+1}$ is done in an alternating way. Each part of the minimization is rather easy because (3.10) is differentiable in $u_{n}$ and the resulting optimality equation

$$
\begin{aligned}
& \left(2 \nu+2 \mu \sum_{j=1, j \neq q}^{N}\left(\uparrow H_{j}\right)^{2}-\lambda \Delta\right) u_{q}^{k+1} \\
= & 2 \nu Z_{q}+\eta \operatorname{div}(\theta)+2 \mu \uparrow H_{q}\left(\sum_{j=1, j \neq q}^{N} u_{j}^{k *} \uparrow H_{j}\right)-\lambda \operatorname{div}\left(d_{q}^{k}-b_{q}^{k}\right) .
\end{aligned}
$$

can approximately be solved by one sweep of Gauss-Seidel. Notice that we decoupled the bands by using $u_{j}^{k *}=u_{j}^{k+1}$ for $j<q$ and $u_{j}^{k *}=u_{j}^{k}$ for $j>q$ on the right hand side of the equation. The solution for $d_{q}^{k+1}$ for a given $u_{q}^{k+1}$ can be given explicitly using the generalized shrinkage formula

$$
\begin{aligned}
\left(d_{q}^{k+1}\right)_{x} & =\max \left(s^{k}-\frac{\gamma}{\lambda}, 0\right) \frac{\nabla_{x} u_{q}^{k+1}+\left(b_{q}^{k}\right)_{x}}{s^{k}}, \\
\left(d_{q}^{k+1}\right)_{y} & =\max \left(s^{k}-\frac{\gamma}{\lambda}, 0\right) \frac{\nabla_{y} u_{q}^{k+1}+\left(b_{q}^{k}\right)_{y}}{s^{k}},
\end{aligned}
$$

with $s^{k}=\sqrt{\left|\nabla_{x} u_{q}^{k+1}+\left(b_{q}^{k}\right)_{x}\right|^{2}+\left|\nabla_{y} u_{q}^{k+1}+\left(b_{q}^{k}\right)_{y}\right|^{2}}$. For more details on the method and its derivation we refer to [24].

3.3. Convergence Speed of Split Bregman minimization. In addition to the results in [24], we want to give the convergence speed of this method, before taking the alternating minimization between $u_{n}^{k+1}$ and $d_{n}^{k+1}$ into account. For a convergence analysis of the alternating minimization we refer to [40].

Our observation holds not only for the application of Split Bregman to anisotropic TV regularization (as in our case), but Split Bregman methods in general, which is why we examine problems of the form

$$
\min _{u}|\phi(u)|_{L_{1}}+R(u, f),
$$


with a quadratic $R$ and a convex regularization term $|\phi(u)|_{L_{1}}$. In our case we have $\phi(u)=\nabla u$ and $R(u, f)$ would be the rest of the energy functional without the TVregularization term. As seen above the idea of Split Bregman is to introduce a new variable $d=\phi(u)$ and solve the resulting constrained problem

$$
\min _{u, d} J(u, d)=\min _{u, d}|d|_{L_{1}}+R(u, f) \text { such that } d=\phi(u)
$$

with Bregman iteration. To be able to give a convergence rate, we need the definition of a source condition.

Definition 3.1 (Source condition). We denote

$$
L(u, d, b)=J(u, d)-\langle b, d-\phi(u)\rangle .
$$

We say that $(\tilde{u}, \tilde{d})$ satisfies a source condition, if there exists a Lagrange multiplier $\tilde{b}$ such that

$$
L(\tilde{u}, \tilde{d}, b) \leq L(\tilde{u}, \tilde{d}, \tilde{b}) \leq L(u, d, \tilde{b}) \quad \forall u, d, b .
$$

We can now state the convergence result.

TheOREM 3.2. Let $(\tilde{u}, \tilde{d})$ be the solution to the problem $\phi(u)=d$. Then $(\tilde{u}, \tilde{d})$ satisfies a source condition and the estimate

$$
D_{J}^{p^{u_{k}}, p^{d_{k}}}\left((\tilde{u}, \tilde{d}),\left(u^{k}, d^{k}\right)\right) \leq \frac{\|\tilde{b}\|}{2 \lambda k}=O\left(\frac{1}{k}\right)
$$

holds, where $\tilde{b}$ is the Lagrange multiplier from the source condition.

The convergence estimate is the result from Theorem 4.1 in [9] for Bregman iteration in general, where the theorem assumes that a source condition is given. Hence, we immediately get a convergence rate for $\left(u^{k}, d^{k}\right)$ towards a solution of the original minimization problem as soon as we can obtain a source condition of the true solution. Our contribution is to show that this is always the case for the split Bregman algorithm.

LEMMA 3.3. The true solution $\tilde{u}$ of the minimization problem (3.15) always satisfies the source condition (3.17).

Proof. The existence of a Lagrange multiplier in our case means that for

$$
L(u, d, b):=|d|_{L_{1}}+R(u, f)-\langle b, d-\phi(u)\rangle
$$

there exists a $\tilde{b}$ such that

$$
L(\tilde{u}, \tilde{d}, \tilde{b}) \leq L(u, d, \tilde{b}) \quad \forall u, d
$$

In other words $(\tilde{u}, \tilde{d})$ has to be the minimizer of the convex functional $L(\cdot, \cdot, \tilde{b})$. That means that a source condition is satisfied if and only if there exists a $\tilde{b}$ such that the following two conditions hold.

1. Optimality condition for $\tilde{d}$

$$
p^{\tilde{d}}-\tilde{b}=0, \quad p^{\tilde{d}} \in \partial|\tilde{d}|_{L_{1}} .
$$

2. Optimality condition for $\tilde{u}$

$$
\tilde{q}+\phi^{*}(\tilde{b})=0, \quad \tilde{q} \in \partial R(\tilde{u}, f) .
$$


The true solution $\tilde{u}$ with $\tilde{d}=\phi(\tilde{u})$ and was a solution to the original minimization problem

$$
\tilde{u}=\arg \min |\phi(u)|+R(u, f),
$$

which mean that it satisfies the optimality condition

$$
\tilde{q}+\tilde{p}=0,
$$

for $\tilde{q} \in \partial R(\tilde{u}, f)$ and $\tilde{p} \in \partial_{u}|\phi(\tilde{u})|$. The latter can be written as $\tilde{p}=\phi^{*}\left(p^{\tilde{d}}\right)$ for a $p^{\tilde{d}} \in \partial_{d}|\tilde{d}|_{L_{1}}$. Therefore, we define $\tilde{b}:=p^{\tilde{d}}$ and immediately obtain the two optimality conditions (3.21) and (3.22), which proves the Lemma.

Equation (3.21) also gives us an estimate on $\tilde{b}$. For a non-zero $\tilde{d}$ we have

$$
\tilde{b}=\frac{\tilde{d}}{|\tilde{d}|}=\operatorname{sign}(\tilde{d})
$$

but even for non differentiable parts where $\tilde{d}$ is zero, we still know that the absolute value of a $p^{\tilde{d}} \in \partial|\tilde{d}|_{L_{1}}$ is bounded by one. Therefore, we get

$$
\tilde{b} \leq|\Omega| \text {. }
$$

Note that this estimate could even be much better if we knew more about the structure of $\tilde{d}$. Since we integrate over a function that we expect to be similar to the sign of $\tilde{d}$ we can hope that a sparse $\tilde{d}$ would give us a much better convergence rate. In our case of using Split Bregman on total variation regularization we have $\tilde{d}=\nabla \tilde{u}$. Thus, a sparse $\tilde{d}$ would correspond to an image with only very few non-zero gradients, e.g. a piecewise constant image. Generally we can conclude that the less non-zero gradients our true, sharpened image has the faster the method will converge. This is of course reasonable since less edges need to be sharpened.

\section{Numerical Results.}

4.1. Results on Multispectral Data. In this section we present fusion results on the Quckbird satellite data, which consists of a four band multispectral image with a spatial resolution of $2.4 \mathrm{~m}$ as well as a panchromatic image with a resolution of $0.6 \mathrm{~m}$. The images were taken at exactly the same time and exactly the same position such that only translational registration needed to be done to align the two images. For the sake of clarity we moved the figures and tables of this section into the appendix.

To evaluate our method we compare it to the standard methods IHS, PCA, Brovey, stationary wavelet fusion and to the other variational method, $\mathrm{P}+\mathrm{XS}$. The comparison is based on seven different image quality metrics that measure spectral quality and one metric measuring spatial quality. The metrics we used are the Relative Dimensionless Global Error in Synthesis (ERGAS) (e.g. used in [22, 5]), the Spectral Angle Mapper (SAM) $([48,22,5])$, Spectral Information Divergence (SID) ([14]), Universal Image Quality Index (Q-average) ([47]), Root Mean Squared Error (RMSE), Relative Average Spectral Error (RASE) ([16]) and Correlation Coefficients (CC) ([45]) as well as the spatial quality measure introduced in [49], which we will refer to as Filtered Correlation Coefficients (FCC).

Our method is specifically designed to preserve the spectral information from the low resolution image. Unfortunately, we do not have a dataset with a multispectral image at different resolution, such that we are missing a dataset with ground truth to 
compare our results with. To show that we preserve the low resolution image spectral characteristics we first compare the sharpened images to the upsampled low resolution images.

Secondly, to simulate a low resolution dataset with ground truth, we subsampled our data and compared the fusion results with the original data. This procedure is common for comparing multispectral fusion algorithms (cf. [46, 5, 45]). However, subsampling the data always includes model assumptions, namely the direct problem of producing the low resolution data given the high resolution data. This is information we do not have for true satellite data. If we had this information we would try to solve the corresponding inverse problem. In literature authors have started to investigate the satellite sensor properties by looking at their modulation transfer functions (e.g. [4]). However, we would like to present a general image fusion method without taking specific sensor information into account, which is why in our experiments we simply used bicubic interpolation for the subsampling.

Figures A.1 and A.2 show the fusion result for different pan sharpening methods. We can see that all methods greatly improve the visual quality of the low resolution image. The standard methods PCA, IHS and Brovey always give visually satisfying results. However, by taking a close look at the color of the fused images and the colors of the low resolution image we can see that these methods tend to change some of the colors. Particularly, the colors of the trees and the color of the swimming pool in image A.1 seem to be slightly off. This is a sign for spectral distortion. To quantify spectral quality we calculated the above mentioned quality metrics with respect to the low resolution image. The results are shown in Tables A.1 and A.2 respectively.

The quality metrics indicate, that PCA, IHS and Brovey have the highest spatial quality, but they also distorted the spectral quality most. The fact that Brovey always satisfies $\mathrm{SAM}=0$ comes from the fact that each band is multiplied with the same factor and therefore SAM never changes. However, the other quality metrics show that Brovey still changes the spectral information.

Our VWP and AVWP fusion results are not as sharp as the above mentioned methods. However, our goal was to sharpen the low resolution image while preserving most of the spectral information. Looking at the image quality metrics we can see that AVWP and VWP fusion results are close to the low resolution image in terms of spectral information. For both images VWP and AVWP have the best values in ERGAS, RASE, RMSE and CC and are second in SAM, SID and QAVE. The contrast increased image with $\eta=1.3 \gamma$ has very sharp edges and high visual quality, but we can see in the quality metrics that we gain the visual quality at the price of loosing some of the spectral information of the low resolution image. Nevertheless these we still get much better values than for the standard methods. Particularly, the idea of minimizing a term to keep the spectral angle at zero, still gives very good SAM and SID metric values.

A little surprising are the metric values for spatial quality. In our opinion the result that $\mathrm{P}+\mathrm{XS}$ clearly has the worst spatial quality can not be confirmed by visual analysis. Furthermore, we would argue that the visual quality of $\mathrm{AVWP}^{*}$ is equal to the top methods, PCA, IHS and Brovey. It seems to be difficult to find a metic that measures the "sharpness" of an image precisely. Particularly, almost any metric that we believe to measure "sharpness" could be used in a variational frame work to minimize for.

As mentioned above, we also simulated fusion data with ground truth by subsampling the given data. Figures A.3, A.4 and A.5 show the fusion results and Tables 
A.3, A.4 and A.5 the corresponding quality metric values. Notice that the images denoted by "ground truth" are now the original images and "low resolution" are the image we obtained by subsampling the "ground truth" using bicubic interpolation. Looking at the quality metrics we can see that although the difference between the methods seems to be smaller, VWP and AVWP still perform very well. The contrast increased AVWP images give much better quality values than in the previous comparison, which shows that the contrast improvement could be of practical relevance. Comparing to the ground truth, the spectral quality of AVWP* does not seem to be bad.

We can see that the low resolution image seems to be far from the true data in terms of absolute pixel values, e.g. in the RMSE and RASE metric. At least for the first test image all methods were able to improve these values in comparison to the low resolution image. However, the SAM value is generally best for the low resolution image, which encourages and strengthens our idea to explicitly constrain this spectral metric in our energy functional. The SAM value of all VWP and AVWP fusion results is the best or close to being the best. The combination of this result and the fact, that VWP could be used to sharpen an arbitrary number of bands, motivates the extension of VWP to hyperspectral image fusion.

4.2. Results on Hyperspectral Data. To demonstrate that our algorithm easily extends to higher dimensional images we worked with two datasets: An 82band AVIRIS hyperspectral image of San Diego Harbor and a 210 band HYDICE hyperspectral image of an urban scene in Texas which is freely available online ([38]). Since the datasets were not accompanied by a master image, we looked at the same scene on Google Maps ${ }^{\mathrm{TM}}$ ([1]) (which offers very high resolution images) and extracted several scenes as our master images. Unfortunately, the hyperspectral images are spatially distorted in comparison to the Google Maps ${ }^{\mathrm{TM}}$ images. For the examples shown in this report we did the required registration on small parts of the image manually. Results of sharpening hyperspectral images are shown in Figure A.6 in the appendix.

In general we can say that the visual quality is greatly increased by the sharpening process. While in the low resolution hyperspectral images small objects can almost not be visually identified, the sharpened images are very close to the master images in terms of spatial quality. We should mention that we do not know when the Google Maps ${ }^{\mathrm{TM}}$ picture was taken. The boxes in the second image of Figure A.6 for instance could be things we see in the master image that were not present in the hyperspectral image and therefore appear as phantoms. One has to be careful with the introduced spatial information, if the two images were not taken at the same time.

As mentioned earlier the most important issue in sharpening hyperspectral images is not to change the spectral signature of during the sharpening process since this information is used for material classification. To investigate how the spectral signature changes we select two pixels in the 82 band hyperspectral scene and look at their spectral signature. Figure A.7 shows the result of the sharpening process as well as the two pixels we selected to examine the change of spectral response during the sharpening process.

The upper left pixel is in the middle of a box where there are no edges or texture even in the master image. At these pixels we match our current iteration to the low resolution image and therefore do not change the intensity in any band. This can be seen in its spectral signature before and after the image fusion. The curves match almost exactly, the signature did not change. At the other pixel we are sure to change 
the signature because we want to increase the contrast and enhance the edge. Our energy model allows this increase of contrast, but enforces the frequency vectors to stay parallel. Investigating the spectral responses we can see that the signatures are different after the sharpening process. However, norming both signature to 1 they again match almost perfectly. The spectral angle is kept at almost zero degrees such that any algorithm base on SAM will perform equally well on the sharpened image as on the low resolution data.

4.3. Comparison of Split Bregman vs. ADI. For our energy minimization problem the most important difference between these methods is the additional regularization parameter $\epsilon$ that is needed by the ADI method to smooth the TV term. It can be shown that the time step for the ADI method is restricted proportional to $\epsilon$. The larger we choose $\epsilon$ the faster ADI will be, but on the other hand we minimize an energy that is different from the original energy. Edges will be smoothed out for too large $\epsilon$.

Split Bregman does not need the additional regularization and minimizes our energy more precisely. However, we still use an $\epsilon$ regularization for the calculation of $\theta_{\epsilon}$. This effect might partly compensate the big advantages the Split Bregman method seems to have for standard TV-denoising as shown in [24].

Our original data multispectral intensity values are in the range between 0 and a a little above 1000. To preserve this precision during the sharpening process, the epsilon regularization should not exceed the reciprocal of the range of values, i.e. $\epsilon \leq 10^{-3}$. Comparing the two minimization methods is difficult, since they minimize slightly different energies, the additionally regularized and the original energy. Figure 4.1 shows the decay of both types of energies for both methods. We can see that Split Bregman minimizes the energy slightly faster. Note that ADIs runtime depends on the choice of $\epsilon$ while Split Bregmans runtime is independent. We can see that the final energy value for ADI is lower than the final Split Bregman energy in the additionally regularized case and vice versa in for the original energy.
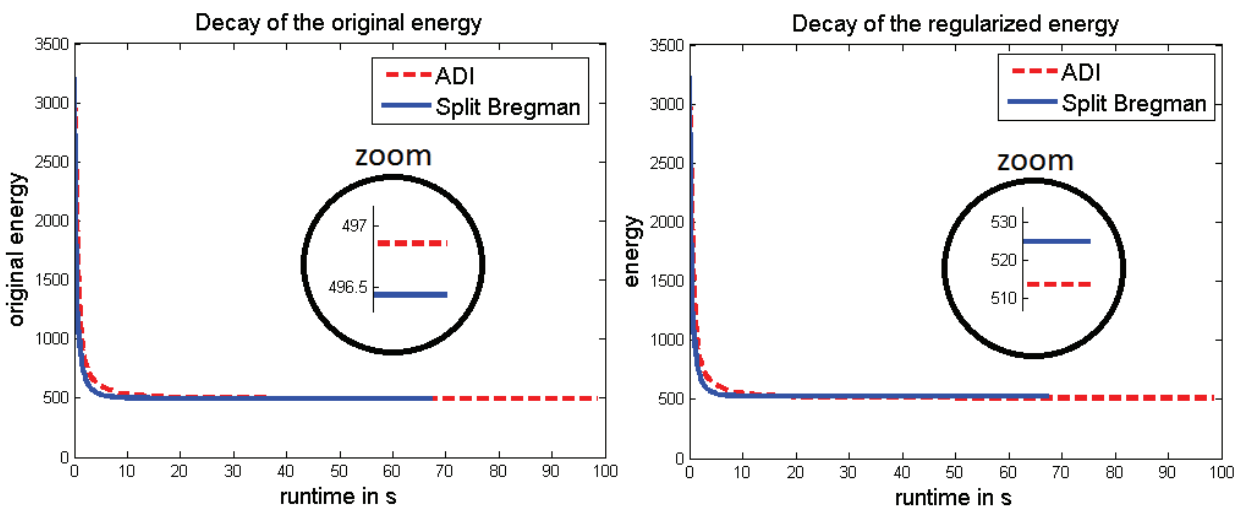

FIG. 4.1. Comparison of ADI and Split Bregman for energy minimization

To get a better and fairer comparison, we rewrite Split Bregman, such that it minimizes the additional regularized energy. However, this is just for comparison purposes, because it significantly slows down the method. Nevertheless we can directly compare the two methods this way. To show the effect of an even smaller epsilon 
(which would for instance be necessary for higher resolved images), we ran the two methods for $\epsilon=10^{-4}$.

Figure 4.2 shows the decay of energy over 2000 iterations on the left. Both methods have a steep drop in energy at the beginning, but ADI slows down and takes many more iterations to reach the same energy as Split Bregman. The minimum energy of Split Bregman is 479.26 while the minimum energy of ADI is 479.31 The Split Bregman energy minimum is not reached at the last iteration. At some point the energy doesn't decrease any more; it slightly increases and than reaches an absolute steady state. This could be due to numerical errors, rounding errors and the fact the we use alternating minimization for the bands, and for the two Split Bregman variables as well as the fact that we solve for these variables only approximately by Gauss-Seidel (for each band) and a fixed point iteration (for the splitted variable $d$ ). However, the difference to the minimal energy is 0.0026 , which accords to an average change of less than $4 \cdot 10^{-8}$ per pixel. This precision is very hard to resolve and would require much more computational afford. We can guess that the true minimal energy might be around 479.2. To be able to resolve the speed of decay better, we use a semi logarithmic plot of the energy divided by the minimal energy minus 1 (see Figure 4.2 right).
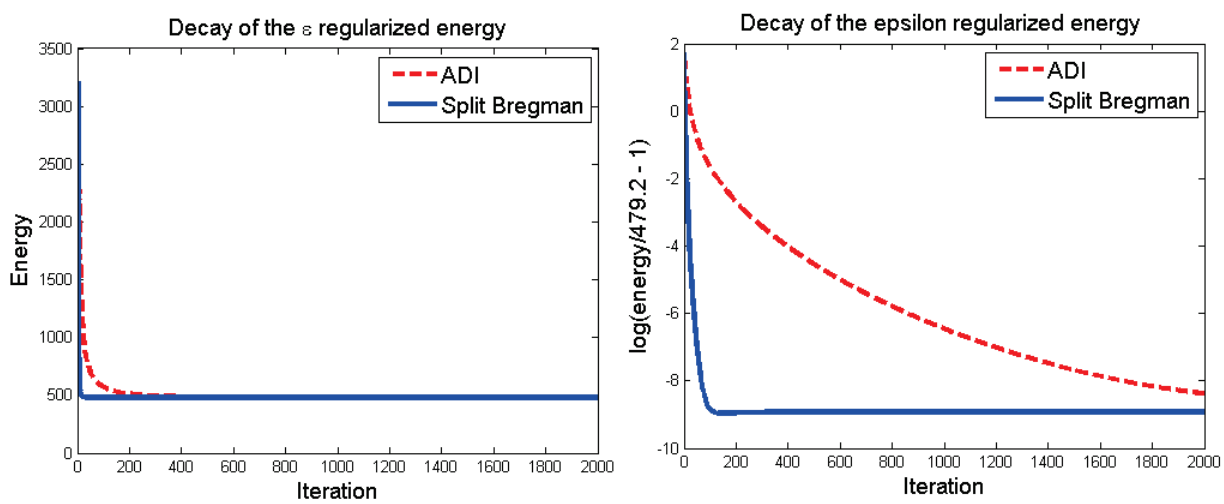

FIG. 4.2. Comparison of ADI and Split Bregman for energy minimization

Split Bregman minimizes the energy really fast and reaches a minimum after about 150 iterations. ADI takes longer, particularly to reach a very high precision solution. However, we should mention that this examination (particularly with $\epsilon=10^{-4}$ ) is of interest for very exact solutions. It becomes more and more important the better the resolution of the sensors gets. For our current data the choice $\epsilon=10^{-3}$ (as examined above) probably is sufficient and for visually satisfying results displayed in RGB colors with values in $[0,255]$ we could even choose $\epsilon=5 * 10^{-3}$.

5. Conclusions. We proposed a variational method for sharpening high dimensional spectral images which extends from usual pan-sharpening to an arbitrary number of bands with a master image that dose not need to be panchromatic. The energy model incorporates the alignment of all unit normal vectors of the level sets of each band with the master image and uses a fidelity term with a combination of the original and a wavelet fused spectral image. By keeping the ratio of all bands constant we assure spectral quality. Large weights on this spectral quality term force the spectral angle between the original and the sharpened spectral image to be close to zero. For 
higher visual quality we chose different weights in the geometry forcing term and to introduce higher edge curvature and therefore higher contrast.

For the optimization we compared a gradient descent ADI method with the Split Bregman algorithm and can conclude that Split Bregman seems to be the superior minimization method. A theoretical convergence speed for Split Bregman was proved.

For future research one could try to incorporate technical information about the satellite sensors into the variational framework and other types of sensors and images could be used. To automate the sharpening process a robust registration method is needed. To reduce the bleeding of colors over some of the edges, deblurring could be included in the sharpening method. Furthermore, this method could be combined with hyperspectral analysis methods like demixing to not only improve the spatial but also the spectral resolution of the image. New detection and classification methods especially suitable for sharpened images could be developed, which take the spectral as well as the spatial information into account. 
Appendix A. Figures and Tables.

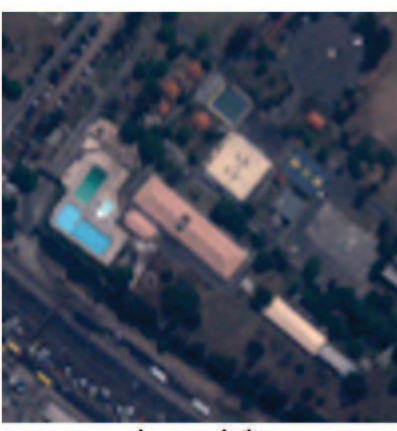

low resolution

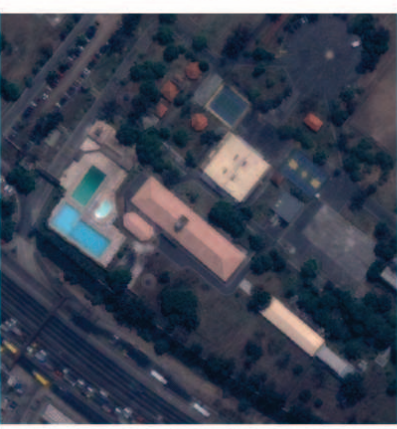

$\mathrm{P}+\mathrm{XS}$

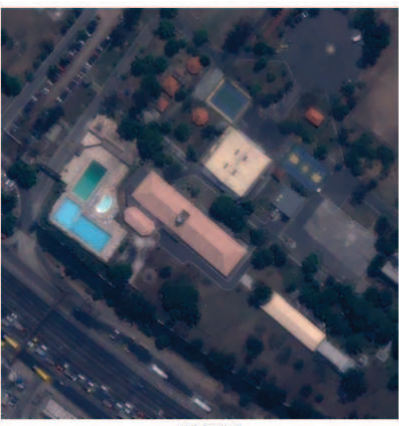

AVWP

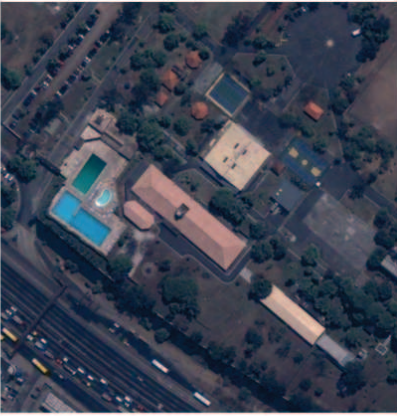

PCA

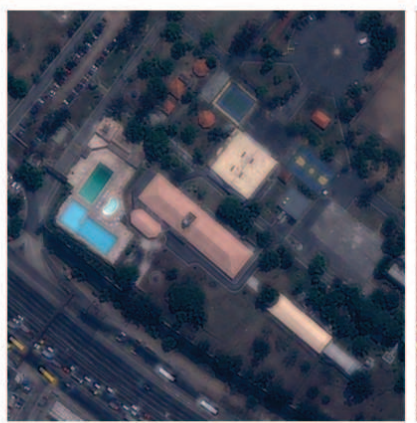

Wavelet

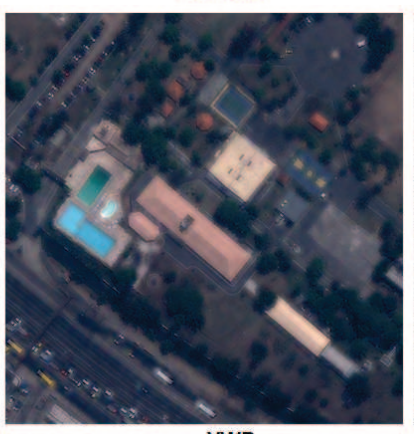

VWP

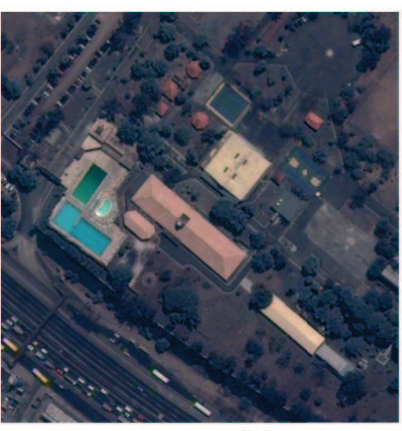

IHS

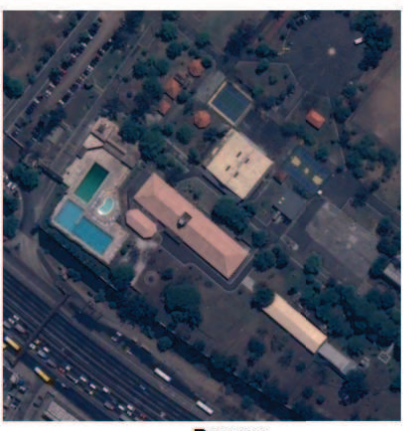

Brovey

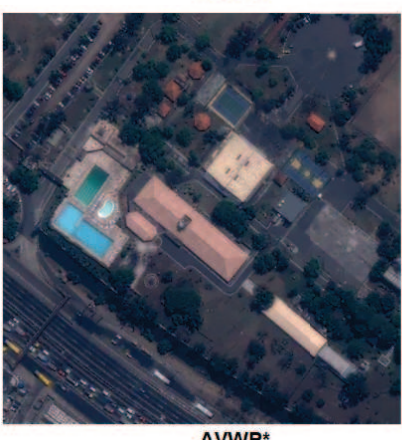

AVWP*

FIG. A.1. Results for sharpening multispectral images

TABLE A.1

Image quality metrics for Figure A.1

\begin{tabular}{|l|r|r|r|r|r|r|r|r|}
\hline & ERGAS & Q-AVE & RASE & RMSE & SAM & \multicolumn{1}{|c|}{ SID } & CC & FCC \\
\hline Brovey & 3.29 & 0.923 & 13.29 & 51.5 & $\mathbf{0 . 0}$ & $\mathbf{0 . 0}$ & 4.11 & $\mathbf{0 . 9 8}$ \\
\hline IHS & 3.35 & 0.917 & 12.69 & 49.15 & 1.239 & 5.54 & 5.14 & $\mathbf{0 . 9 8}$ \\
\hline PCA & 3.29 & 0.938 & 12.96 & 50.23 & 1.427 & 5.57 & 3.69 & $\mathbf{0 . 9 8}$ \\
\hline Wavelet & 1.96 & 0.976 & 7.87 & 30.51 & 0.716 & 0.46 & 1.48 & 0.97 \\
\hline P+XS & 1.87 & 0.982 & 7.38 & 28.60 & 1.353 & 0.99 & 2.30 & 0.84 \\
\hline AVWP & 2.52 & 0.961 & 10.14 & 39.28 & 0.510 & 0.18 & 3.18 & 0.91 \\
\hline AVWP & 1.60 & $\mathbf{0 . 9 8 4}$ & 6.44 & 24.95 & 0.546 & 0.19 & 1.24 & 0.91 \\
\hline VWP & $\mathbf{1 . 5 9}$ & $\mathbf{0 . 9 8 4}$ & $\mathbf{6 . 3 5}$ & $\mathbf{2 4 . 6 1}$ & 0.222 & 0.05 & $\mathbf{1 . 0 8}$ & 0.90 \\
\hline
\end{tabular}




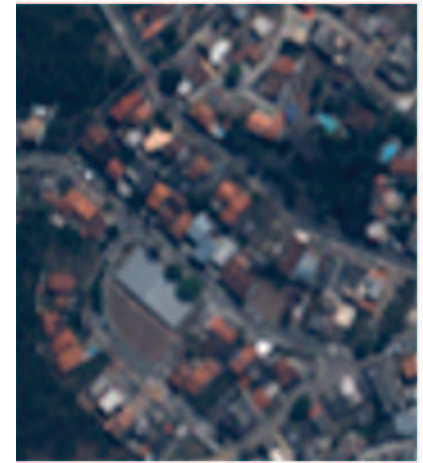

low resolution

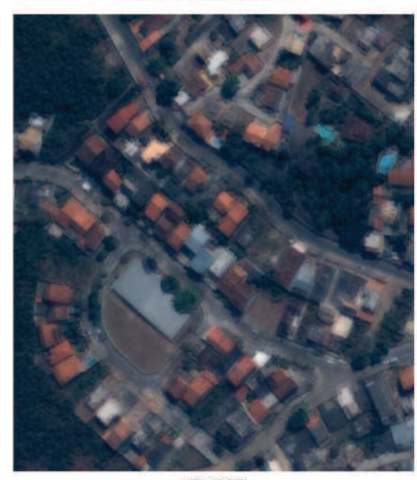

P+XS

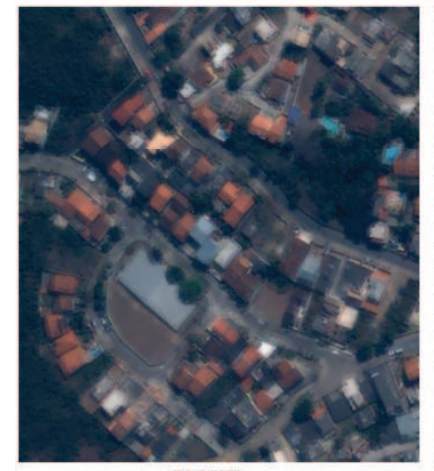

AVWP

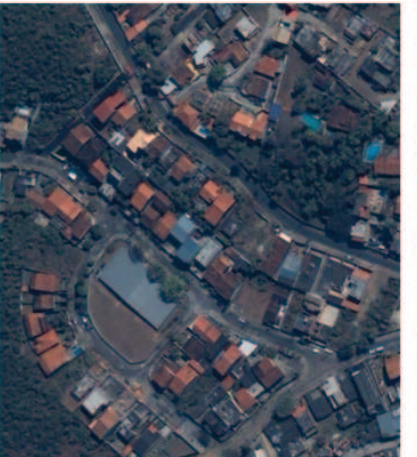

PCA

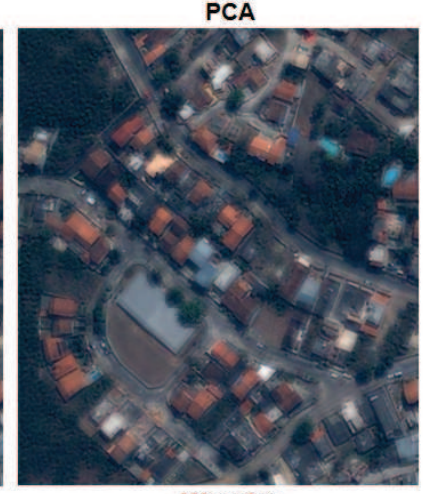

Wavelet

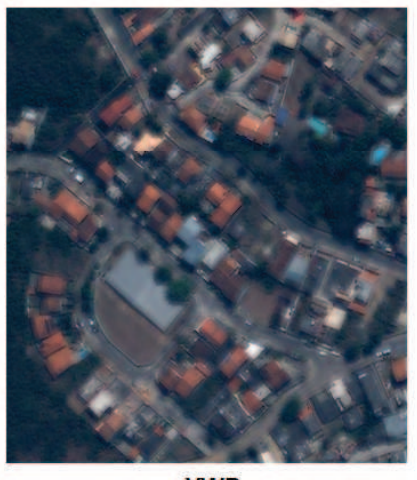

VWP

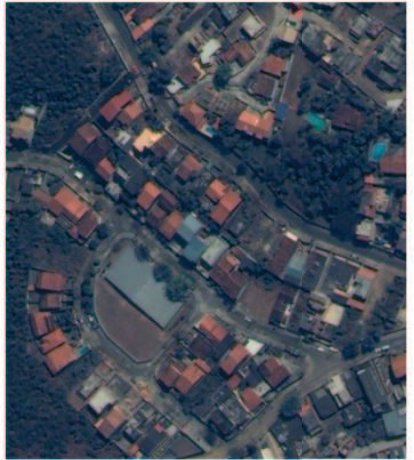

IHS

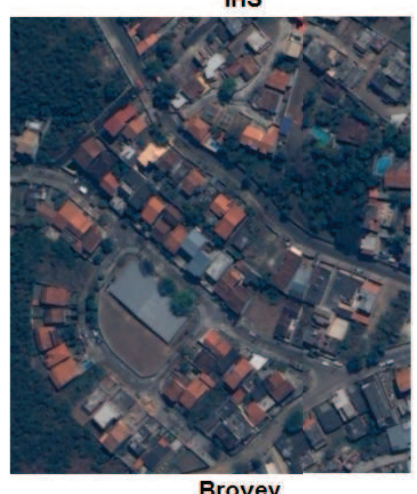

Brovey

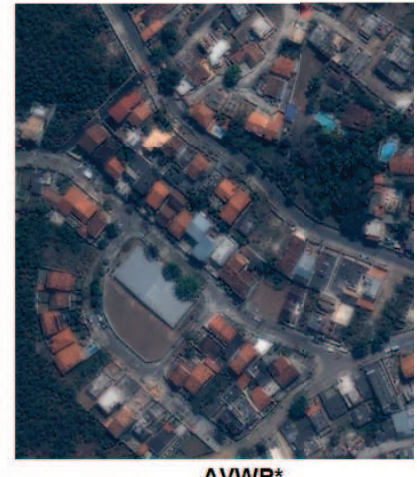

AVWP*

FIG. A.2. Results for sharpening multispectral images 
TABLE A.2

Image quality metrics for Figure A.2

\begin{tabular}{|l|r|r|r|r|r|r|r|r|}
\hline & ERGAS & Q-AVE & RASE & RMSE & SAM & \multicolumn{1}{|c|}{ SID } & CC & FCC \\
\hline Brovey & 3.45 & 0.877 & 13.99 & 51.69 & $\mathbf{0 . 0}$ & $\mathbf{0 . 0}$ & 3.79 & $\mathbf{0 . 9 8}$ \\
\hline IHS & 3.60 & 0.862 & 13.57 & 50.16 & 1.338 & 1.33 & 6.02 & $\mathbf{0 . 9 8}$ \\
\hline PCA & 3.83 & 0.874 & 14.65 & 54.13 & 2.168 & 3.24 & 6.54 & 0.97 \\
\hline Wavelet & 2.23 & 0.954 & 8.88 & 32.83 & 0.726 & 0.42 & 3.11 & 0.97 \\
\hline P+XS & 2.17 & 0.966 & 8.41 & 31.09 & 1.329 & 0.94 & 2.84 & 0.84 \\
\hline AVWP & 2.94 & 0.922 & 11.85 & 43.8 & 0.420 & 0.12 & $\mathbf{0 . 6 0 7}$ & 0.91 \\
\hline AVWP & 1.81 & 0.968 & 7.28 & 26.92 & 0.467 & 0.14 & 2.03 & 0.91 \\
\hline VWP & $\mathbf{1 . 7 0}$ & $\mathbf{0 . 9 7 2}$ & $\mathbf{6 . 8 1}$ & $\mathbf{2 5 . 1 5}$ & 0.232 & 0.04 & 2.31 & 0.90 \\
\hline
\end{tabular}

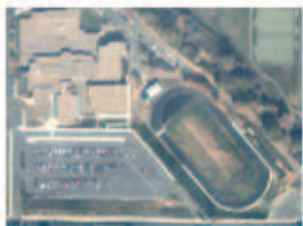

ground truth

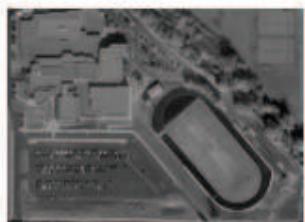

pancromatic

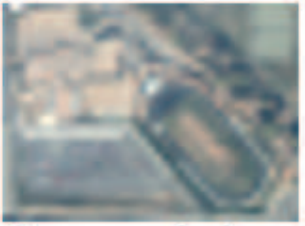

low resolution

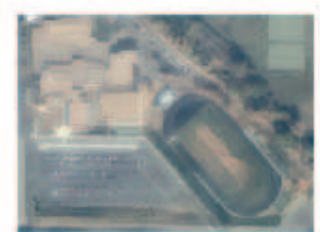

P+XS

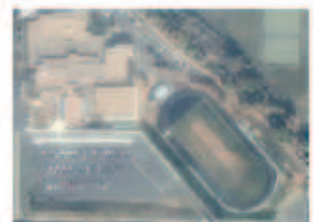

AVWP

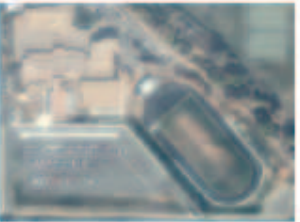

PCA

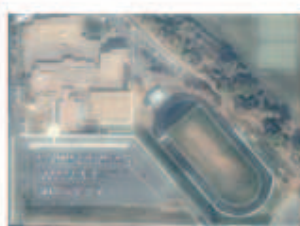

Wavelet

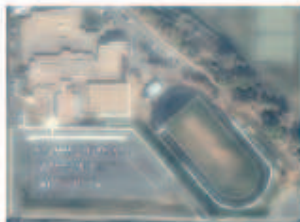

VWP

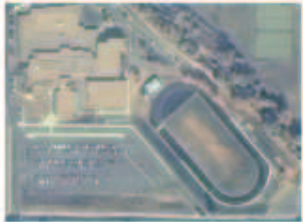

IHS

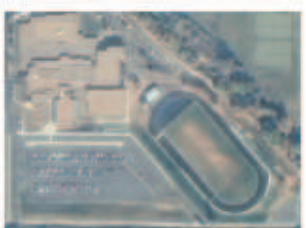

Brovey

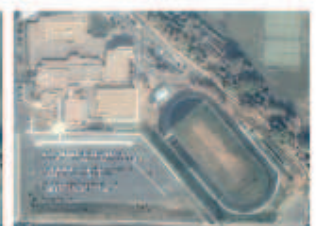

AVWP*

FIG. A.3. Results for sharpening multispectral images 
TABLE A.3

Image quality metrics for Figure A.3

\begin{tabular}{|l|r|r|r|r|r|r|r|r|}
\hline & ERGAS & Q-AVE & RASE & RMSE & SAM & \multicolumn{1}{|c|}{ SID } & CC & FCC \\
\hline Brovey & 2.58 & 0.876 & 10.54 & 28.20 & 3.00 & 5.01 & 4.56 & $\mathbf{0 . 9 6}$ \\
\hline IHS & 2.73 & 0.856 & 10.91 & 29.19 & 3.40 & 6.26 & 5.98 & $\mathbf{0 . 9 6}$ \\
\hline PCA & 2.74 & 0.880 & 11.74 & 31.40 & 3.69 & 6.30 & 2.26 & 0.83 \\
\hline Wavelet & 2.47 & 0.907 & 10.40 & 27.82 & 3.19 & 5.62 & $\mathbf{1 . 8 9}$ & 0.95 \\
\hline P+XS & 3.22 & 0.879 & 12.53 & 33.52 & 3.02 & $\mathbf{4 . 8 3}$ & 4.00 & 0.81 \\
\hline AVWP & $\mathbf{2 . 3 7}$ & $\mathbf{0 . 9 1 4}$ & $\mathbf{9 . 8 3}$ & $\mathbf{2 6 . 3 1}$ & $\mathbf{2 . 9 9}$ & 4.97 & 3.61 & 0.92 \\
\hline AVWP & 2.43 & 0.908 & 10.20 & 27.27 & 3.00 & 4.98 & 3.48 & 0.89 \\
\hline VWP & $\mathbf{2 . 3 7}$ & $\mathbf{0 . 9 1 4}$ & 9.98 & 26.71 & 3.04 & 5.13 & 2.61 & 0.92 \\
\hline Lowres & 3.26 & 0.83 & 13.61 & 36.40 & 3.00 & 5.01 & 7.92 & 0.49 \\
\hline
\end{tabular}

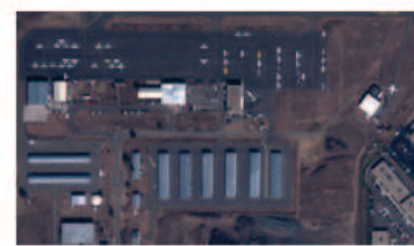

ground truth

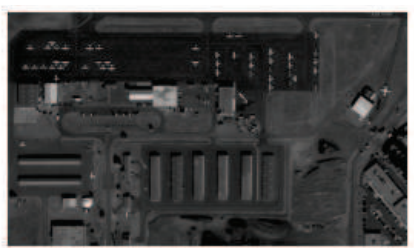

pancromatic

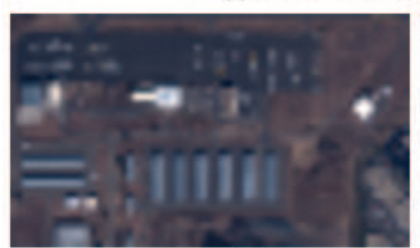

low resolution

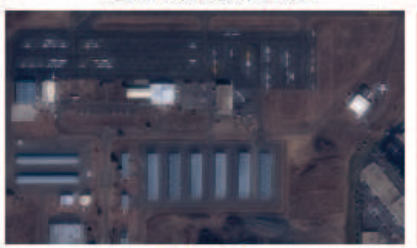

P+XS

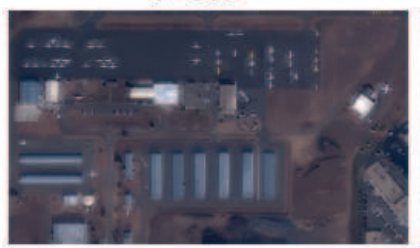

AVWP

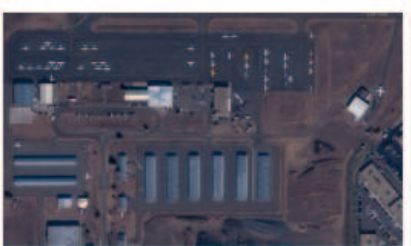

PCA

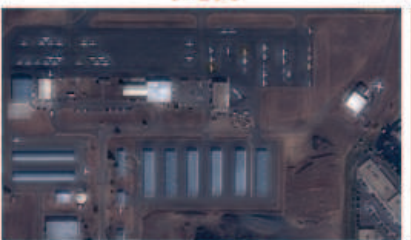

Wavelet

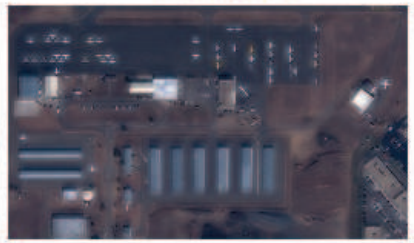

VWP

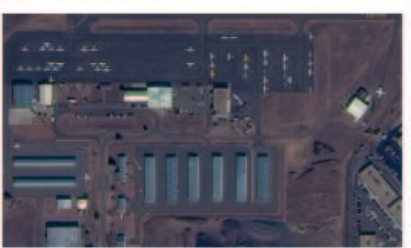

IHS

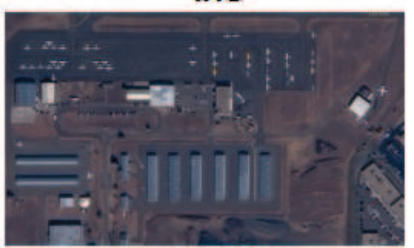

Brovey

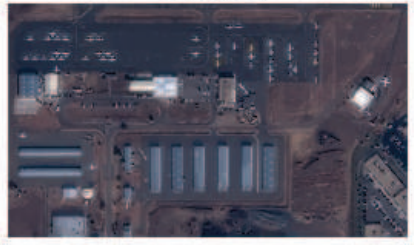

AVWP*

FIG. A.4. Results for sharpening multispectral images 


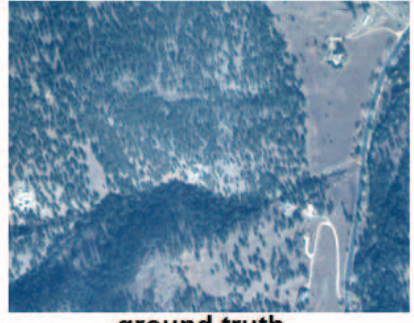

ground truth

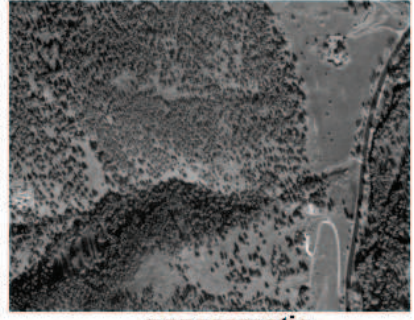

pancromatic

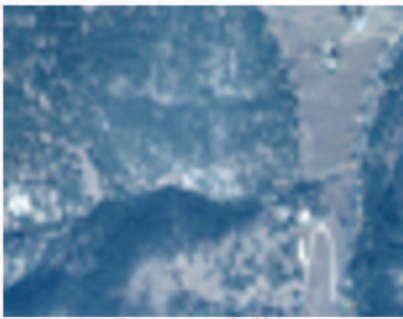

low resolution

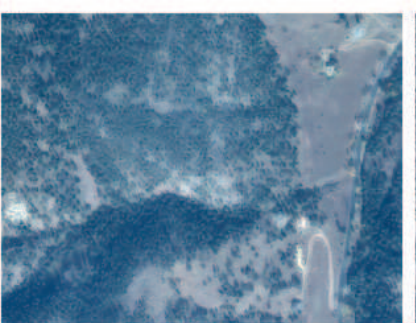

$\mathrm{P}+\mathrm{XS}$

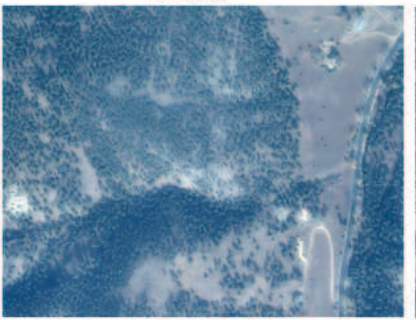

AVWP

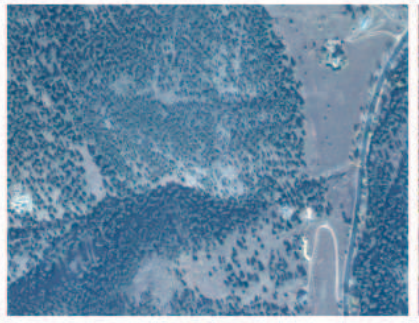

PCA

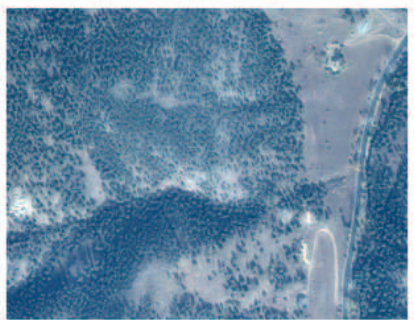

Wavelet

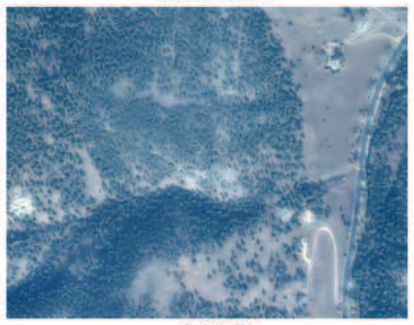

VWP

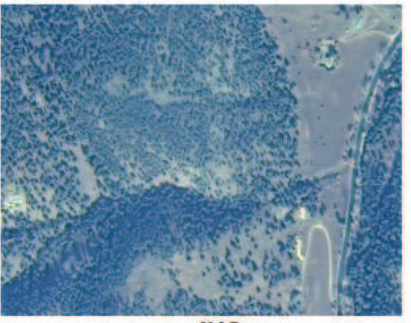

IHS

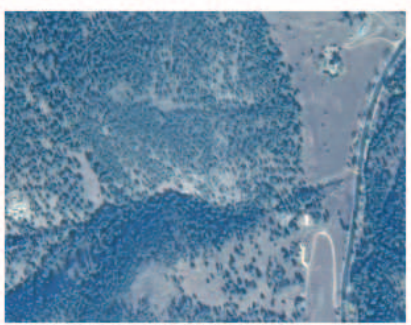

Brovey

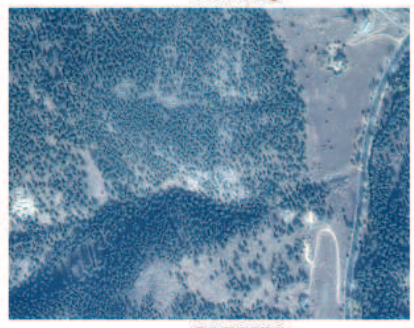

AVWP*

FiG. A.5. Results for sharpening multispectral images 
TABLE A. 4

Image quality metrics for Figure A.4

\begin{tabular}{|l|r|r|r|r|r|r|r|r|}
\hline & ERGAS & Q-AVE & RASE & RMSE & SAM & \multicolumn{1}{|c|}{ SID } & CC & FCC \\
\hline Brovey & 3.61 & 0.843 & 14.63 & 39.91 & $\mathbf{2 . 2 3}$ & $\mathbf{3 . 0 1}$ & 0.92 & 0.98 \\
\hline IHS & 3.69 & 0.834 & 14.65 & 39.95 & 2.52 & 3.74 & 3.30 & $\mathbf{0 . 9 9}$ \\
\hline PCA & 3.56 & 0.851 & 14.54 & 39.67 & 2.27 & 3.06 & 2.31 & $\mathbf{0 . 9 9}$ \\
\hline Wavelet & 3.20 & 0.889 & 13.10 & 35.74 & 2.42 & 3.48 & 1.36 & 0.98 \\
\hline P+XS & 3.47 & 0.889 & 13.56 & 36.97 & 2.47 & 3.37 & 0.98 & 0.86 \\
\hline AVWP & 3.38 & 0.882 & 13.77 & 37.57 & 2.24 & 3.02 & 3.85 & 0.94 \\
\hline AVWP & 3.04 & 0.897 & 12.47 & 34.01 & 2.25 & 3.03 & $\mathbf{0 . 7 2}$ & 0.94 \\
\hline VWP & $\mathbf{2 . 9 3}$ & $\mathbf{0 . 9 0 7}$ & $\mathbf{1 2 . 0 3}$ & $\mathbf{3 2 . 8 2}$ & 2.27 & 3.10 & 0.83 & 0.94 \\
\hline Lowres & 3.41 & 0.867 & 13.99 & 38.15 & $\mathbf{2 . 2 3}$ & $\mathbf{3 . 0 1}$ & 2.44 & 0.44 \\
\hline
\end{tabular}

TABLE A.5

Image quality metrics for Figure A.5

\begin{tabular}{|l|r|r|r|r|r|r|r|r|}
\hline & ERGAS & Q-AVE & RASE & RMSE & SAM & \multicolumn{1}{|c|}{ SID } & CC & FCC \\
\hline Brovey & 3.93 & 0.756 & 17.25 & 35.54 & $\mathbf{4 . 5 1}$ & $\mathbf{9 . 8 0}$ & 9.23 & 0.98 \\
\hline IHS & 4.27 & 0.713 & 17.94 & 36.94 & 5.29 & 13.77 & 8.92 & 0.98 \\
\hline PCA & 3.97 & 0.798 & 17.27 & 35.58 & 4.70 & 10.88 & 6.96 & 0.97 \\
\hline Wavelet & 3.80 & 0.822 & 16.90 & 34.81 & 4.72 & 10.86 & 9.54 & $\mathbf{0 . 9 9}$ \\
\hline P+XS & 3.94 & $\mathbf{0 . 8 4 5}$ & 17.16 & 35.34 & 4.52 & 9.86 & 7.97 & 0.88 \\
\hline AVWP & 4.37 & 0.757 & 18.97 & 39.06 & 4.52 & 9.84 & 10.86 & 0.95 \\
\hline AVWP & $\mathbf{3 . 6 0}$ & 0.828 & $\mathbf{1 6 . 4 0}$ & $\mathbf{3 3 . 7 7}$ & 4.52 & 9.85 & 8.31 & 0.96 \\
\hline VWP & 3.72 & 0.818 & 16.83 & 34.67 & 4.54 & 9.97 & 8.42 & 0.95 \\
\hline Lowres & 3.78 & 0.823 & 17.93 & 36.92 & $\mathbf{4 . 5 1}$ & $\mathbf{9 . 8 0}$ & $\mathbf{6 . 9 4}$ & 0.34 \\
\hline
\end{tabular}
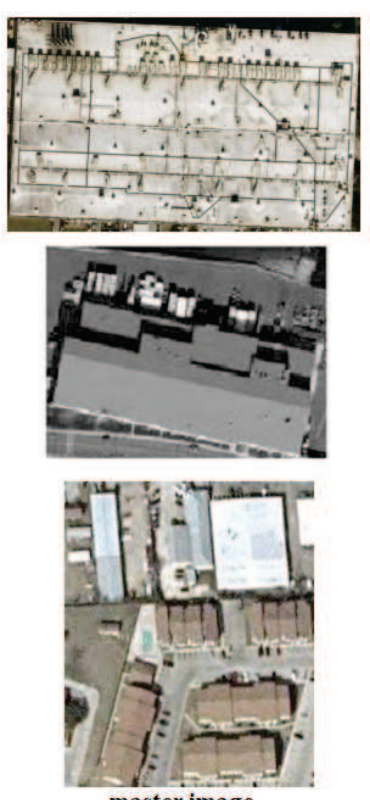

master image
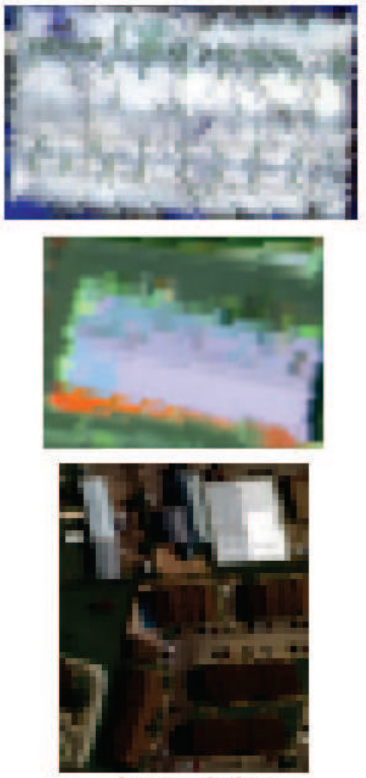

low resolution
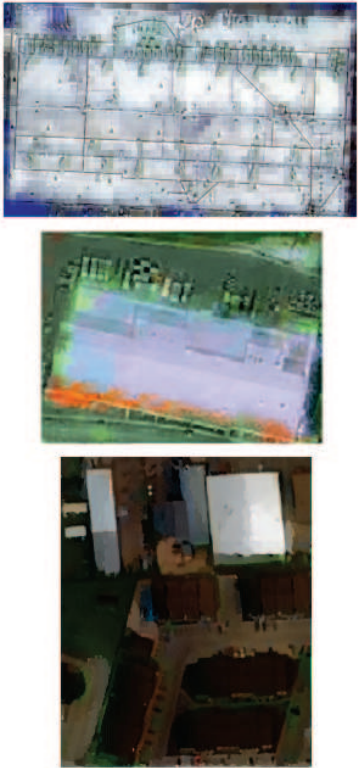

sharpened

FIG. A.6. Results for sharpening hyperspectral images 

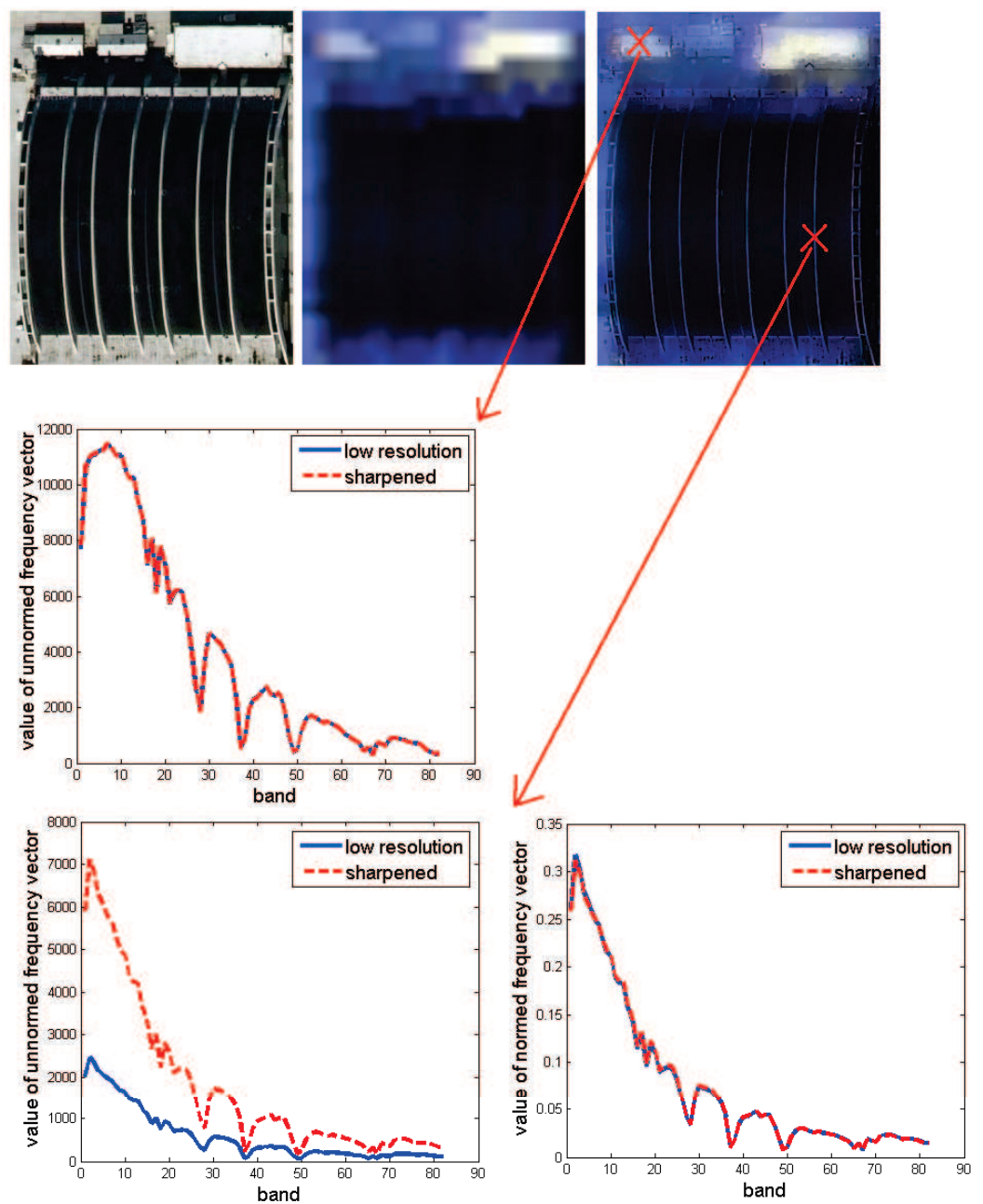

FIG. A.7. Behavior of the spectral signature during the sharpening process

Acknowledgements. The authors would like to thank Melissa Strait, Sheida Rahmani and Daria Merkurev for their help and especially for provinding the Matlab code for all image quality metrics. We would further like to thank Julia Dobrosotskaya, Stanley Osher, Jarome Darbon and Ernest Esser for their advice.

\section{REFERENCES}

[1] Google, Inc., Google Maps. Online: http://maps.google.com, Jan. 2009.

[2] B. Aiazzi, L. Alparone, S. Baronti, and A. Garzelli, Context-driven fusion of high spatial and spectral resolution images based on oversampled multiresolution analysis, IEEE Transactions on Geoscience and Remote Sensing, 40 (2002), pp. 2300 - 2312.

[3] B. Aiazzi, L. Alperone, S. Baronti, I. Pippi, And M. Selva, Generalized Laplacian pyramidbased fusion of $M S+P$ image data with spectral distortion minimization, ISPRS Commission III, Symposium 2002, (2002).

[4] L. Alparone, B. Ainzzi, S. Baronti, and A. Garzelli, A global quality measurement of pansharpened multispectral imagery, 2003 IEEE International Geoscience and Remote Sensing Symposium. Proceedings., 1 (2003), pp. 458-460. 
[5] L. Alparone, L. Wald, J. Chanussot, C. Thomas, P. Gamba, and L. M. Bruce, Comparison of pansharpening algorithms: Outcome of the 2006 GRS-S data-fusion contest, IEEE Transactions on Geoscience and Remote Sensing, 45 (2007), pp. 3012-3021.

[6] C. Ballester, V. Caselles, L. Igual, and J. Verdera, A variational model for P+XS image fusion, International Journal of Computer Vision, 69 (2006), pp. $43-58$.

[7] L. M. BREgman, The relaxation method of finding the common point of convex sets and its application to the solution of problems in convex programming, USSR Computational Mathematics and Mathematical Physics, 7 (1967), pp. $200-217$.

[8] M. Burger And S. Osher, Convergence rates of convex variational regularization, Inverse Problems, 20 (2004), pp. 1411 - 1421.

[9] M. Burger, E. Resmerita, And L. He, Error estimation for Bregman iterations and inverse scale space methods in image restoration, Computing, 81 (2007), pp. $109-135$.

[10] J.-F. CAI, S. OSher, And Z. Shen, Fast linearized Bregman iteration for compressed sensing and sparse denoising, UCLA CAM Reprots, 08-37 (2008).

[11] D. Calvetti And L. Reichel, Application of ADI iterative methods to the restoration of noisy images, SIAM J. Matrix Anal. Appl., 17 (1996), pp. 165-186.

[12] YoungJoon Cha And Seonguai Kim, Edge-forming methods for image zooming, Journal of Mathematical Imaging and Vision, 25 (2006), pp. 353-364.

[13] T. F. Chan, J. Shen, And H.-M. Zhou, A total variation wavelet inpainting model with multilevel fitting parameters, Congress, Advanced signal processing algorithms, architectures, and implementations XVI, 69 (2006).

[14] C.-I Chang, Spectral information divergence for hyperspectral image analysis, Geoscience and Remote Sensing Symposium, 1999. IGARSS '99 Proceedings. IEEE 1999 International, 1 (1999), pp. $509-511$.

[15] Y. Chibani AND A. HouAcine, Redundant versus orthogonal wavelet decomposition for multisensor image fusion, Pattern Recognition, 36 (2003), pp. 879 - 887.

[16] M. CHOI, A new intensity-hue-saturation fusion approach to image fusion with a tradeoff parameter, IEEE Transactions of Geoscience and Remote Sensing, 44 (2006), pp. 1672 1682 .

[17] M. ChOI, H.-C. KIM, N. I. CHO, AND H. O. KIM, An improved intensity-huesaturation method for IKONOS image fusion, submitted to IJRS. Online: http://amath.kaist.ac.kr/research/paper/06-9.pdf.

[18] S.D. Conte And C. DEBoor, Elementary Numerical Analysis, McGraw-Hill, 1972.

[19] J. Dobrosotskaya And A. L. Bertozzi, A wavelet-Laplace variational technique for image deconvolution and inpainting, IEEE Transactions on Image Processing, 17 (2008), pp. 657 -663 .

[20] J. Douglas And D. W. Peaceman, Numerical solution of two-dimensional heat-flow problems, AIChE Journal, 1 (1955), pp. 505 - 512.

[21] Q. Du, O. Gungor, And J. Shan, Performance evaluation for pan-sharpening techniques, Geoscience and Remote Sensing Symposium, 2005. IGARSS '05. Proceedings. 2005 IEEE International, 6 (2005), pp. $4264-4266$.

[22] Q. Du, N. H. Younan, R. King, And V. P. Shah, On the performance evaluation of pansharpening techniques, IEEE Geoscience and Remote Sensing Letters, 4 (2007), pp. 518 522 .

[23] A. Garzelli And F. Nencini, Fusion of panchromatic and multispectral images by genetic algorithms, IEEE International Conference on Geoscience and Remote Sensing Symposium, 2006., (2006), pp. 3810-3813.

[24] T. Goldstein And S. OsheR, The split Bregman method for $L_{1}$ regularized problems, SIAM Journal on Imaging Sciences, 2 (2009).

[25] M. Gonzalez-Audicana, X. Otazu, O. Fors, and J. Alvarez-Mozos, A low computationalcost method to fuse IKONOS images using the spectral response function of its sensors, IEEE Transactions on Geoscience and Remote Sensing, 44 (2006), pp. 1683- 1691.

[26] G. Hong AND Y. ZHAng, The effects of different types of wavelets on image fusion, International Conference on Image Processing, 1995. Proceedings., 3 (1995), pp. $248-251$.

[27] Kisee Joo And SEOngJai Kim, PDE-based image restoration, II: Numerical schemes and color image denoising, tech. report, 2003.

[28] R. L. KInG AND J. WANG, A wavelet based algorithm for pan-sharpening Landsat 7 imagery, Geoscience and Remote Sensing Symposium, 2001. IGARSS '01. IEEE 2001 International, 2 (2001), pp. $849-851$.

[29] O. M. Lysaker, S. Osher, And X.-C. TAI, Noise removal using smoothed normals and surface fitting, IEEE Transaction on Image Processing, 13 (2004), pp. 1345 - 1357.

[30] F. MALGOUYRES, Mathematical analysis of a model which combines total variation and wavelet 
for image restoration, Journal of information processes, 2 (2002), pp. 1 - 10.

[31] N. Memarsadeghi, J. Le Moigne, and D. Mount, Image fusion using cokriging, Geoscience and Remote Sensing Symposium, 2006. IGARSS 2006. IEEE International Conference on, (2006), pp. $2518-2521$.

[32] M. MöLler, A Variational Approach to Sharpening High Dimensional Images, master's thesis, Westfaelische Wilhelms-Universitaet Muenster, Germany, June 2009.

[33] M. Möller, T. Wittman, And A. L. Bertozzi, A variational approach to hyperspectral image fusion, Proc. SPIE Conference on Algorithms and Technologies for Multispectral, Hyperspectral, and Ultraspectral Imagery XV, (2009).

[34] S. Osher, M. Burger, D. Goldfarb, J. Xu, and W. Yin, An iterative regularization method for total variation-based image restoration, SIAM Multiscale Modeling and Simulation, 4 (2005), pp. $460-489$.

[35] X. Otazu, M. Gonzalez-Audicana, O. Fors, and J. Nunez, Introduction of sensor spectral response into image fusion methods. application to wavelet-based methods, IEEE Transactions on Geoscience and Remote Sensing, 10 (2005), pp. 2376- 2385.

[36] X. Otazu, M. Gonzlez-Audcana, O. Fors, and J. Nez, Introduction of sensor spectral response into image fusion methods. application to wavelet-based methods, IEEE Transactions on Geoscience and Remote Sensing, 43 (2005).

[37] D. W. Peaceman and JR. H. H. Rachford, The numerical solution of parabolic and elliptic differential equations, Journal of the Society for Industrial and Applied Mathematics, 3 (1955), pp. $28-41$.

[38] Air University Space Primer. Chapter 12: Multispectral imagery. Online: http://www.au.af.mil/au/awc/space/primer/multispectral_imagery.pdf, Jan. 2009 .

[39] E. Resmerita AND O. Scherzer, Error estimates for non-quadratic regularization and the relation to enhancing, Inverse Problems, 22 (2006), pp. 801-814.

[40] S. SETZER, Split Bregman algorithm, Douglas-Rachford splitting and frame shrinkage, in SSVM '09: Proceedings of the Second International Conference on Scale Space and Variational Methods in Computer Vision, Berlin, Heidelberg, 2009, Springer-Verlag, pp. 464-476.

[41] V. P. Shah, N. H. Younan, And R. L. King, An efficient pan-sharpening method via a combined adaptive PCA approach and contourlets, IEEE Transactions on Geoscience and Remote Sensing, 46 (2008), pp. 1323-1335.

[42] V. K. ShetTigara, A generalized component substitution technique for spatial enhancement of multispectral images using a higher resolution data set, Photogrammetric engineering and remote sensing, 58 (1992), pp. $561-567$.

[43] C. Thomas, T. Ranchin, L. Wald, and J. Chanussot, Synthesis of multispectral images to high spatial resolution: a critical review of fusion methods based on remote sensing physics, (2008).

[44] T.-M. Tu, P. S. Huang, C.-L. Hung, And C.-P. Chang, A fast intensity-hue-saturation fusion technique with spectral adjustment for IKONOS imagery, IEEE Geoscience and Remote Sensing Letters, 1 (2004).

[45] V. ViJayaraj, C. G. O HARA, And N. H. Younan, Quality analysis of pansharpened images, Geoscience and Remote Sensing Symposium, 2004. IGARSS '04. Proceedings. 2004 IEEE International, 1 (2004).

[46] L. Wald, T. Ranchin, AND M. Mangolini, Fursion of satellite images of different spatial resolutions: Assessing the quality of resulting images, Photogrammetric Engineering and Remote Sensing, 63 (1997), pp. 691-699.

[47] Z. WANG AND A. C. BoviK, A universal image quality index, IEEE Signal Processing Letters, 9 (2002), pp. $81-84$.

[48] R. H. Yuhas, A. F. H. Goetz, and J. W. Boardman, Discrimination among semi-arid landscape endmembers using the spectral angle mapper(SAM) algorithm, Proceeding Summeries 3rd Annual JPL Airborne Geosciense Workshop, (1992), pp. 147 - 149.

[49] J. Zhou, D. L. Civico, and J. A. Silander, A wavelet transform method to merge landsat $T M$ and SPOT panchromatic data, International Journal of Remote Sensing, 19 (1998). 